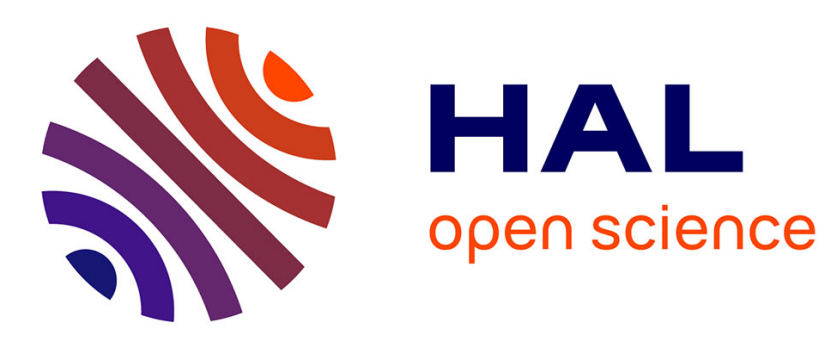

\title{
Efficacy of epetraborole against Mycobacterium abscessus is increased with norvaline
}

Jaryd R Sullivan, Andréanne Lupien, Elias Kalthoff, Claire Hamela, Lorne Taylor, Kim A Munro, T Martin Schmeing, Laurent Kremer, Marcel A Behr

\section{- To cite this version:}

Jaryd R Sullivan, Andréanne Lupien, Elias Kalthoff, Claire Hamela, Lorne Taylor, et al.. Efficacy of epetraborole against Mycobacterium abscessus is increased with norvaline. PLoS Pathogens, 2021, 17 (10), pp.e1009965. 10.1371/journal.ppat.1009965 . inserm-03381888

\section{HAL Id: inserm-03381888 https://www.hal.inserm.fr/inserm-03381888}

Submitted on 18 Oct 2021

HAL is a multi-disciplinary open access archive for the deposit and dissemination of scientific research documents, whether they are published or not. The documents may come from teaching and research institutions in France or abroad, or from public or private research centers.
L'archive ouverte pluridisciplinaire HAL, est destinée au dépôt et à la diffusion de documents scientifiques de niveau recherche, publiés ou non, émanant des établissements d'enseignement et de recherche français ou étrangers, des laboratoires publics ou privés. 


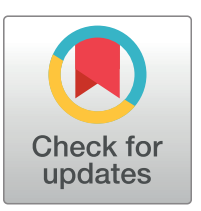

\section{G open ACCESS}

Citation: Sullivan JR, Lupien A, Kalthoff E, Hamela C, Taylor L, Munro KA, et al. (2021) Efficacy of epetraborole against Mycobacterium abscessus is increased with norvaline. PLoS Pathog 17(10): e1009965. https://doi.org/10.1371/journal. ppat.1009965

Editor: Helena Ingrid Boshoff, National Institutes of Health, UNITED STATES

Received: July 6, 2021

Accepted: September 23, 2021

Published: October 12, 2021

Peer Review History: PLOS recognizes the benefits of transparency in the peer review process; therefore, we enable the publication of all of the content of peer review and author responses alongside final, published articles. The editorial history of this article is available here: https://doi.org/10.1371/journal.ppat.1009965

Copyright: @ 2021 Sullivan et al. This is an open access article distributed under the terms of the Creative Commons Attribution License, which permits unrestricted use, distribution, and reproduction in any medium, provided the original author and source are credited.

Data Availability Statement: All relevant data are within the paper and its Supporting Information files. Structures have been deposited on PDB under accession codes 7N11 and 7N12. Sequence
RESEARCH ARTICLE

\section{Efficacy of epetraborole against} Mycobacterium abscessus is increased with norvaline

Jaryd R. Sullivan $\circledast^{1,2,3}$, Andréanne Lupien $\circledast^{2,3}$, Elias Kalthoff $\oplus^{4,5}$, Claire Hamela $\oplus^{6}$, Lorne Taylor $^{7}$, Kim A. Munro ${ }^{4,5}$, T. Martin Schmeing ${ }^{4,5}$, Laurent Kremer $\oplus^{6,8}$, Marcel A. $\operatorname{Behr} \mathbb{D}^{1,2,3,9 *}$

1 Department of Microbiology \& Immunology, McGill University, Montréal, Canada, 2 Infectious Diseases and Immunity in Global Health Program, Research Institute of the McGill University Health Centre, Montréal, Canada, 3 McGill International TB Centre, Montréal, Canada, 4 Department of Biochemistry, McGill University, Montréal, Canada, 5 Centre de Recherche en Biologie Structural, McGill University, Montréal, Canada, 6 Centre National de la Recherche Scientifique UMR 9004, Institut de Recherche en Infectiologie de Montpellier (IRIM), Université de Montpellier, Montpellier, France, 7 Clinical Proteomics Platform, Research Institute of the McGill University Health Centre, Montréal, Canada, 8 INSERM, IRIM, Montpellier, France, 9 Department of Medicine, McGill University Health Centre, Montréal, Canada

* marcel.behr@mcgill.ca

\section{Abstract}

Mycobacterium abscessus is the most common rapidly growing non-tuberculous mycobacteria to cause pulmonary disease in patients with impaired lung function such as cystic fibrosis. $M$. abscessus displays high intrinsic resistance to common antibiotics and inducible resistance to macrolides like clarithromycin. As such, $M$. abscessus is clinically resistant to the entire regimen of front-line $M$. tuberculosis drugs, and treatment with antibiotics that do inhibit $M$. abscessus in the lab results in cure rates of $50 \%$ or less. Here, we identified epetraborole (EPT) from the MMV pandemic response box as an inhibitor against the essential protein leucyl-tRNA synthetase (LeuRS) in $M$. abscessus. EPT protected zebrafish from lethal $M$. abscessus infection and did not induce self-resistance nor against clarithromycin. Contrary to most antimycobacterials, the whole-cell activity of EPT was greater against $M$. abscessus than $M$. tuberculosis, but crystallographic and equilibrium binding data showed that EPT binds LeuRS ${ }_{\text {Mabs }}$ and LeuRS $_{\text {Mtb }}$ with similar residues and dissociation constants. Since EPT-resistant $M$. abscessus mutants lost LeuRS editing activity, these mutants became susceptible to misaminoacylation with leucine mimics like the non-proteinogenic amino acid norvaline. Proteomic analysis revealed that when $M$. abscessus LeuRS mutants were fed norvaline, leucine residues in proteins were replaced by norvaline, inducing the unfolded protein response with temporal changes in expression of GroEL chaperonins and Clp proteases. This supports our in vitro data that supplementation of media with norvaline reduced the emergence of EPT mutants in both $M$. abscessus and $M$. tuberculosis. Furthermore, the combination of EPT and norvaline had improved in vivo efficacy compared to EPT in a murine model of $M$. abscessus infection. Our results emphasize the effectiveness of EPT against the clinically relevant cystic fibrosis pathogen $M$. abscessus, and these findings also suggest norvaline adjunct therapy with EPT could be beneficial for $M$. abscessus and other mycobacterial infections like tuberculosis. 
data has been deposited in the NCBI Sequence Read Archive under the NCBI BioProject ID PRJNA756101. (https://www.ncbi.nlm.nih.gov/sra/ $\mathrm{X)}$.

Funding: This work was funded by Cystic Fibrosis Canada (to J.R.S. and M.A.B.), the Research Institute of the McGill University Health Centre (to J.R.S.), the Harrison Watson Fellowship (to J.R.S.), the Structure-guided Drug Discovery Coalition (to M.A.B.), ClHR operating grant (to M.A.B.) and was supported by Vaincre la Mucoviscidose (RF20200502678) (to L.K) and the Association Gregory Lemarchal (to L.K). The funders had no role in study design, data collection and analysis, decision to publish, or preparation of the manuscript.

Competing interests: The authors have declared that no competing interests exist.

\section{Author summary}

Current antimycobacterial drugs are inadequate to handle the increasing number of nontuberculous mycobacteria infections that eclipse tuberculosis infections in many developed countries. Of particular importance for cystic fibrosis patients, Mycobacterium abscessus is notoriously difficult to treat where patients spend extended time on antibiotics with cure rates comparable to extreme drug resistant $M$. tuberculosis. Here, we identified epetraborole (EPT) with in vitro and in vivo activities against M. abscessus. We showed that EPT targets the editing domain of the leucyl-tRNA synthetase (LeuRS) and that escape mutants lost LeuRS editing activity, making these mutants susceptible to misaminoacylation with leucine mimics. Most importantly, combination therapy of EPT and norvaline limited the rate of EPT resistance in both M. abscessus and M. tuberculosis, and this was the first study to demonstrate improved in vivo efficacy of EPT and norvaline compared to EPT in a murine model of $M$. abscessus pulmonary infection. The demonstration of norvaline adjunct therapy with EPT for M. abscessus infections is promising for cystic fibrosis patients and could translate to other mycobacterial infections, such as tuberculosis.

\section{Introduction}

Mycobacterium abscessus is a nontuberculous mycobacterium that commonly causes chronic lung disease, especially among patients with cystic fibrosis [1]. Treatment of pulmonary exacerbation relies on a regimen of intravenous amikacin (AMK), tigecycline, and imipenem plus an oral macrolide if the isolate is susceptible to macrolides [2,3]. Despite guideline-based treatment, combination therapy typically results in cure rates of $50 \%$ or less due to the bacterium being intrinsically resistant to many antibiotic classes [4,5]. Unfortunately, the M. abscessus drug-development pipeline is limited to a handful of repurposed drugs (clofazimine, rifabutin, and bedaquiline) [6]. Lately, antibiotics that have shown activity in pre-clinical studies include the oxazolidinones (LCB01-0371 [7] and tedizolid [8]) that target the 50S ribosomal subunit and PIPD1/indol-2-carboxamides that target the mycolic acid transporter MmpL3 $[9,10]$.

Recently, aminoacyl-tRNA synthetases (aaRSs) became targets of interest for drug discovery when benzoxaboroles were identified as novel boron-based pharmacophores $[11,12]$. aaRSs are enzymes that aminoacylate tRNAs with their cognate amino acids. All aaRSs have an aminoacylation domain while some are bifunctional and contain an editing domain. The aminoacylation domain forms an aminoacyl adenylate through condensation of the amino acid with ATP, and then transfers the aminoacyl moiety to the 3 ' terminal adenosine of the tRNA acceptor stem. Some aaRSs, however, have evolved an editing domain to ensure the correct amino acid is ligated to its tRNA [13,14]. This domain is critical for aaRSs that must distinguish their cognate amino acid from structurally similar amino acids like branched-chain amino acids. Leucyl-tRNA synthetases (LeuRSs) are examples of aaRSs that rely on their editing domains to limit misaminoacylation of tRNA with near-cognate amino acids and maintain the fidelity of the genetic code.

Herein, we identified epetraborole (EPT) from the Medicines for Malaria Venture (MMV) Open pandemic response box as a LeuRS inhibitor in M. abscessus with nanomolar activity in vitro and activity in zebrafish embryos and NOD.SCID mice. Interestingly, EPT was more active against $M$. abscessus than $M$. tuberculosis, and this observation was not supported by 
structural differences in the crystal structures of the LeuRS editing domain of M. abscessus and M. tuberculosis bound to EPT. Furthermore, we highlighted the utility of norvaline to target LeuRS editing deficient escape mutants and suppress resistance in vitro by misincorporation of norvaline in place of leucine residues resulting in disrupted protein folding. Importantly, we showed that EPT and norvaline combination has improved efficacy over EPT monotherapy in a murine model of M. abscessus infection. These data support the potential of the benzoxaborole scaffold in the antimycobacterial drug pipeline and suggest that its activity can be potentiated by supplementation with norvaline or a norvaline derivative.

\section{Results}

\section{Discovery of an antimycobacterial inhibitor against M. abscessus}

To identify compounds with antimycobacterial activity, we first engineered a luminescent $M$. abscessus ATCC 19977 strain which constitutively expressed the luxCDABE operon [15] (M. abscessus lux). We then screened the 176 compound open-library provided by GlaxoSmithKline (S1A-S1C Fig) and the 400 compound Pathogen and Pandemic Response Boxes from MMV (S1D-S1F Fig) using a threshold $\geq 90 \%$ reduction in luminescence compared to nontreated bacteria at $10 \mu \mathrm{M}$. Primary hits (20 compounds) were tested in a secondary screen using the resazurin microtiter assay (REMA) at $10 \mu \mathrm{M}$ on the M. abscessus ATCC 19977 strain. Secondary hits (9 compounds) were then tested in a dose-response assay on M. abscessus ATCC 19977 from a fresh solid compound to determine MIC ${ }_{90}$. The HepG2 (human hepatocytes) cell line was used to assess the toxicity of hits. Among three compounds ( $0.3 \%$ positive hits of 976 compounds) from the pandemic response box that satisfied primary and secondary criteria, we identified EPT (Fig 1A) as having the most potent antimycobacterial activity and highest therapeutic index (Table $1, \mathrm{MIC}_{90} 0.23 \mu \mathrm{M}\left(0.063 \mu \mathrm{g} / \mathrm{mL}\right.$ ), TI $\left(\mathrm{TD}_{50} / \mathrm{MIC}_{90}\right)$ EPT > 330). It was previously reported in $M$. tuberculosis that potent in vitro growth inhibitors could display carbon-source-dependent effects, which leads to a loss of activity when advanced into in vivo models [16]. We thus measured the $\mathrm{MIC}_{90}$ of EPT on different carbon sources (glycerol vs acetate), in the presence or absence of the detergent Tween-80, and in different nutrient bases (Middlebrook 7H9 vs cation-adjusted Mueller-Hinton). EPT was active in all assayed growth conditions but lost potency in cation-adjusted Mueller Hinton (CaMH) media (S1 Table). Lower activity of EPT in CaMH media is unsurprising as it was previously shown that rifamycins [17] as well as other antimicrobials [18] lose activity in CaMH media $(0.5 \mathrm{mg} / \mathrm{L}$ $\mathrm{Ca}^{2+}$ and $0.05 \mathrm{mg} / \mathrm{L} \mathrm{Mg}^{2+}$ in $7 \mathrm{H} 9 ; 20-25 \mathrm{mg} / \mathrm{L} \mathrm{Ca}^{2+}$ and $10-12.5 \mathrm{mg} / \mathrm{L} \mathrm{Mg}^{2+}$ in CaMH). It is thought that some antimicrobials may chelate divalent metal ions, thus limiting their uptake in cells.

Next, we tested the in vitro activity of EPT against a panel of M. abscessus clinical isolates belonging to the three subspecies of the M. abscessus complex (M. abscessus, M. massiliense, and $M$. bolletii) with smooth and rough colony morphologies and with different drug susceptibility profiles to various antibiotics. There was no loss in activity against clinical isolates (range of $0.014-0.046 \mu \mathrm{g} / \mathrm{mL}$ ) nor different morphologies (S2 Table). To determine the spectrum of activity of EPT, we curated a panel of various mycobacteria and representative gram-positive and gram-negative bacteria. Interestingly, EPT appears to be more selective for M. abscessus with lower activity against $M$. tuberculosis $\mathrm{H} 37 \mathrm{Rv}$ (S3 Table). In vitro growth kill kinetics indicated that EPT is bacteriostatic against $M$. abscessus as previously demonstrated against $M$. tuberculosis [19], while $10 \%$ of CFUs were lost at 24 hours with AMK and rifampicin (RIF) at $20 \mathrm{X} \mathrm{MIC}{ }_{90}$. Interestingly, these two antimycobacterial agents with bactericidal action against other mycobacteria lose this ability when targeting M. abscessus (Fig 1B) [20]. 
A<smiles>NC[C@H]1OB(O)c2c(OCCCO)cccc21</smiles>

C

24h
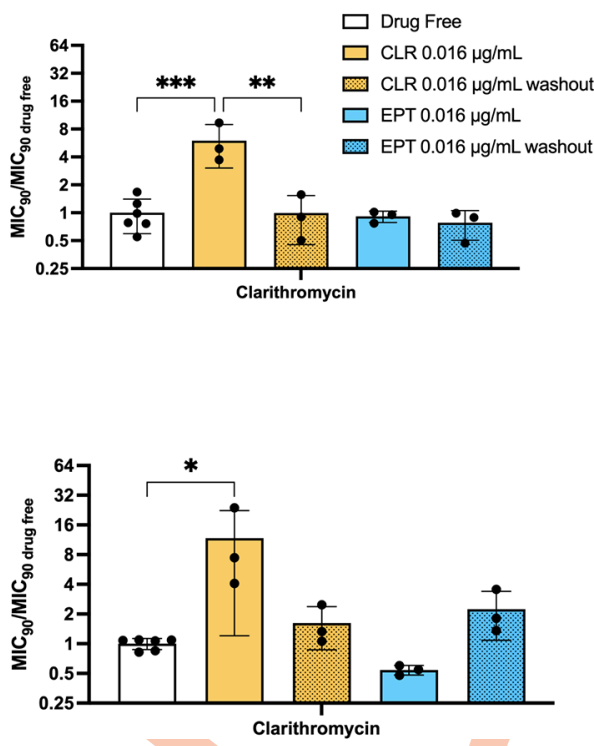

B

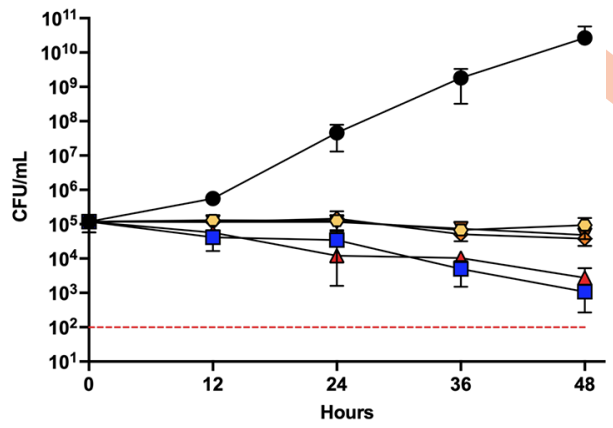

$14 d$
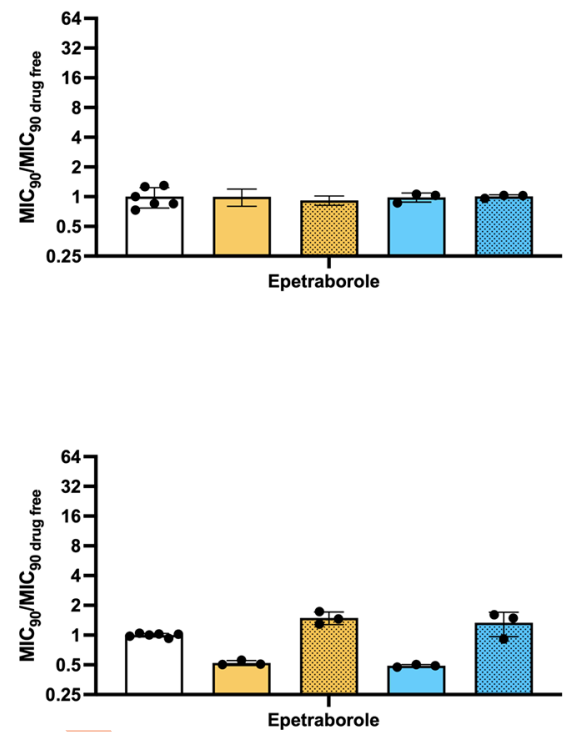

Fig 1. EPT in vitro activity against M. abscessus. A Structure of EPT. B Epetraborole kill kinetics of M. abscessus in vitro. Bacteria were incubated in $7 \mathrm{H} 9$ complete with drug-free (black circles), $\mathrm{AMK} 20 \mathrm{X} \mathrm{MIC} 90$ (blue squares, $80 \mu \mathrm{g} /$ $\mathrm{mL}$ ), RIF 20X MIC 90 (red triangles, $60 \mu \mathrm{g} / \mathrm{mL}$ ), EPT 20X MIC 90 (yellow hexagons, $1.6 \mu \mathrm{g} / \mathrm{mL}$ ), EPT 40X MIC 90 (orange diamonds, $3.2 \mu \mathrm{g} / \mathrm{mL}$ ), or EPT 80X MIC 90 (brown inverted triangles, $6.4 \mu \mathrm{g} / \mathrm{mL}$ ). The dashed red line indicates $99.9 \%$ or $3 \log _{10}$ reduction in CFU/mL threshold of a bactericidal drug. Data shown is mean $\pm \mathrm{SD}$ of three independent experiments. $\mathrm{C} \mathrm{MIC}_{90}$ from induced cultures relative to drug free conditions. Bacteria were grown to $\mathrm{OD}_{600} 0.05$ and induced with no drug (white), $0.016 \mu \mathrm{g} / \mathrm{mL} \mathrm{CLR}$ (yellow), $0.016 \mu \mathrm{g} / \mathrm{mL}$ EPT (blue). After 24 hours (left) or 14 days (right) of induction, cultures were passaged for 6 days in drug free media (dotted bars). Data shown is mean $\pm \mathrm{SD}$ from three independent experiments. Error bars for drug free data derived from $\mathrm{MIC}_{90} / \mathrm{MIC}_{90}$ average from three or six replicates. ${ }^{*}, \mathrm{p}=0.02 ;^{* *}, \mathrm{p}=0.0017 ;^{* * * *}, \mathrm{p}=0.0005$ by one-way ANOVA with Tukey's multiple comparisons test.

https://doi.org/10.1371/journal.ppat.1009965.g001 
Table 1. MMV Open pandemic response box hits and reference compounds.

\begin{tabular}{l|l|l|l|l|l}
\hline \multirow{2}{*}{ Parameter } & & & Compound & BDQ \\
\cline { 2 - 6 } & EPT & ERV & IQN & 3.3 & 0.67 \\
\hline $\mathrm{MIC}_{90}(\mu \mathrm{g} / \mathrm{mL})$ & 0.063 & 1.9 & $>63$ & $>39$ & $9.4^{\mathrm{a}}$ \\
\hline $\mathrm{TD}_{50}(\mu \mathrm{g} / \mathrm{mL})$ & $>27$ & 33 & 12 & 14 & 18 \\
\hline $\mathrm{TI}\left(\mathrm{TD}_{50} / \mathrm{MIC}\right.$ & & 30 \\
\hline $\mathrm{MW}(\mathrm{g} / \mathrm{mol})$ & $>430$ & 631.52 & 393.40 & 555.51 & 4.5 \\
\hline
\end{tabular}

EPT, Epetraborole; ERV, Eravacycline; IQN, Isoquinoline urea; BDQ, Bedaquiline; AMK, Amikacin; $\mathrm{MIC}_{90}$, concentration of drug that inhibits $90 \%$ of bacterial growth; $\mathrm{TD}_{50}$, concentration of drug with $50 \%$ toxicity against HepG2 cell line. TI, therapeutic index.

${ }^{\mathrm{a}}$ Data from Lupien, A et al. Antimicrob Agents Chemother. 2018

\section{Clinical considerations for EPT}

Macrolides represent the cornerstone of M. abscessus therapy. However, macrolide susceptibility in vitro does not correlate with clinical outcome success due to point mutations at positions 2058 or 2059 in the $23 \mathrm{~S}$ rRNA $\mathrm{rrl}$ gene (E. coli numbering), and inducible macrolide resistance conferred from the recently identified ribosomal methyltransferase erm(41) [21,22]. Nash et al (2009) discovered that M. abscessus exhibited an inducible resistant phenotype to macrolides, like clarithromycin (CLR), during a 14-day incubation. To this extent, we asked if EPT induced self-resistance or cross-resistance to macrolides. To answer this question, we performed the inducible macrolide resistance assay where M. abscessus was cultured with a subinhibitory concentration of CLR $(0.016 \mu \mathrm{g} / \mathrm{mL})$ or EPT $(0.016 \mu \mathrm{g} / \mathrm{mL})$ for 14 days. At 24 hours and 14 days, aliquots of culture were collected, and the MIC 90 of CLR and EPT was determined using REMA. Relative to drug-free conditions, $M$. abscessus cultured with CLR displayed an increased $\mathrm{MIC}_{90}$ to CLR while the EPT MIC $\mathrm{C}_{90}$ for bacteria exposed to EPT remained unchanged up to 14 days (Fig $1 \mathrm{C}$ solid bars). To ensure the increased $\mathrm{MIC}_{90}$ resulted from inducible resistance rather than the selection of spontaneous CLR and EPT resistant mutants, the cultures were passaged in antibiotic-free media for 6 additional days and the $\mathrm{MIC}_{90}$ measurements were repeated. In the case of CLR resistance, the $\mathrm{MIC}_{90}$ returned to baseline after the antibiotics were removed (Fig $1 \mathrm{C}$ dotted bars). In addition, EPT stimulation did not result in cross-resistance to CLR, and CLR induction did not result in cross-resistance to EPT. The latter is an important finding with clinical significance because CLR is known to impart resistance to aminoglycoside antibiotics [23].

Because M. abscessus infections require 18-24 months of antibiotic courses, multidrug treatments are standard practice $[2,3]$. To ensure EPT would not hamper a multidrug regimen, we performed checkerboard assays with common antimycobacterial drugs. In the checkerboard assays, we used RIF/CLR and CLR/AMK as the synergy and antagonism controls, respectively $[23,24]$. We did not observe antagonism between EPT and various antimycobacterial agents that target a range of cellular processes (S2 Fig). Whether EPT should be included in a multidrug regimen remains to be determined.

\section{EPT protects zebrafish from $M$. abscessus infection}

In order to investigate the in vivo activity of EPT, we used the embryonic zebrafish model of $M$. abscessus infection, which has been developed to test the in vivo efficacy of drugs against $M$. abscessus [25-27]. Initial experiments indicated that EPT concentrations up to $40 \mu \mathrm{g} / \mathrm{mL}$ (final concentration in fish water) did not interfere with larval development and was well tolerated in embryos when treatment was applied for up to 5 days with daily drug renewal (Fig 2A). 
A

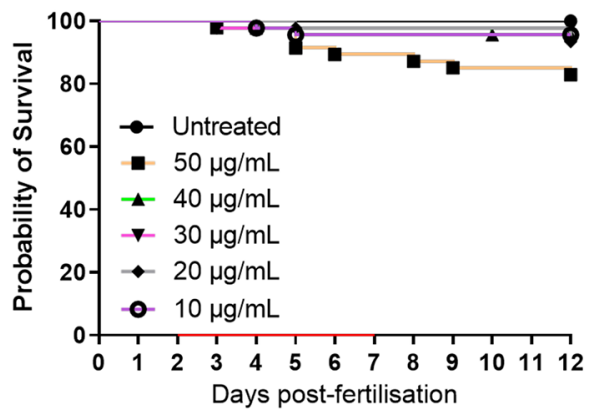

C

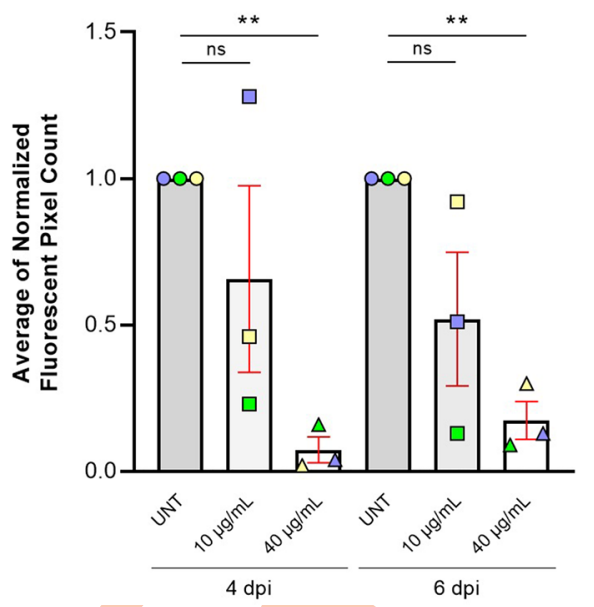

B

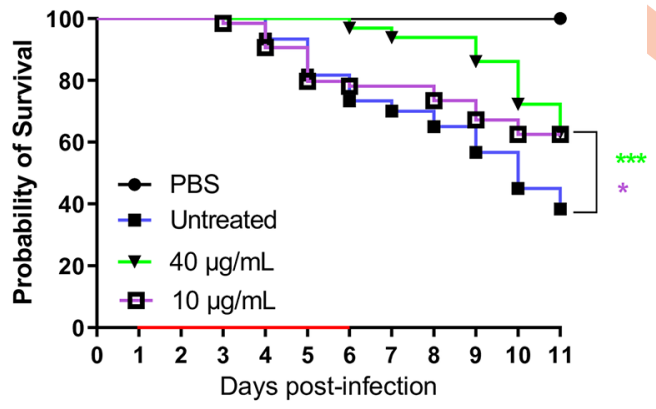

D

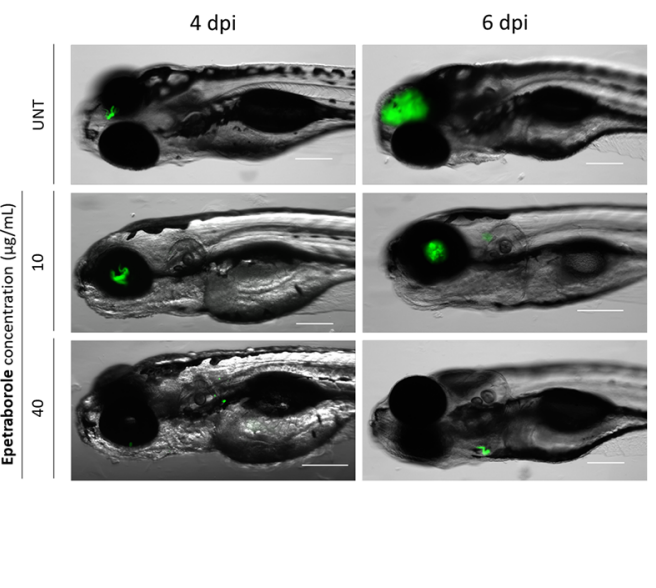

Fig 2. EPT in vivo activity against M. abscessus. A EPT toxicity against zebrafish embryos. Groups of uninfected embryos were immersed in water containing 10 to $50 \mu \mathrm{g} / \mathrm{mL}$ EPT for 5 days. Red bar indicates duration of treatment. Data is from two independent experiments. B EPT in vivo activity in zebrafish embryos. Zebrafish embryos at $30 \mathrm{~h}$ postfertilization were infected with approximately 200 CFUs of M. abscessus expressing green fluorescent wasabi or PBS via caudal vein injection. At $1 \mathrm{dpi}$, embryos were randomly split into approximately 20 embryos per group and exposed to increasing concentrations of EPT $(10$ and $40 \mu \mathrm{g} / \mathrm{mL})$ in fish water. Drugs were renewed at a daily basis for 5 days (red bar) after which embryos were washed twice in fresh embryo water and maintained in fish water. Each treatment group was compared against the untreated infected group with significant differences calculated using the log-rank (Mantel-Cox) statistical test for survival curves. Data shown are the merge of four independent experiments. ${ }^{*}, \mathrm{p}=0.02{ }^{* * *}, \mathrm{p}=0.0007$. C Fluorescent Pixel Counts (FPC) determination using the ImageJ software, reflecting the bacterial loads at 4 and 6 days post-infection (dpi). Each bar represents a pool of the average of normalized FPC from three independent experiments ( $n=22$ to 36 embryos). Error bars represent standard deviations. Statistical significance was determined using Welch's $t$ test. ${ }^{* *}, \mathrm{p} \leq 0.0054$. D Representative whole embryos from the untreated group (upper panels) and treated group (10 or $40 \mu \mathrm{g} / \mathrm{mL} \mathrm{EPT;} \mathrm{lower} \mathrm{panels)} \mathrm{at} 4$ and $6 \mathrm{dpi}$. Green overlay represents M. abscessus expressing wasabi. Scale bar, $200 \mu \mathrm{m}$.

https://doi.org/10.1371/journal.ppat.1009965.g002 
Green fluorescent wasabi-expressing $M$. abscessus ( $\mathrm{R}$ variant) was microinjected in the caudal vein of embryos at 30 hours post-fertilization. EPT was directly added at 1 day post-infection to the water containing the infected embryos, with EPT-supplemented water changed on a daily basis for 5 days. Embryo survival was monitored and recorded daily for 11 days. While a slight increase in the survival rate was observed with $10 \mu \mathrm{g} / \mathrm{mL}$ EPT, this effect was significantly improved with $40 \mu \mathrm{g} / \mathrm{mL}$ EPT with a delay in larval mortality, as compared to the untreated group (Fig $2 \mathrm{~B}$ ). The protection provided by EPT is maintained throughout the 5-day treatment course and was correlated with decreased bacterial burdens beginning $4 \mathrm{dpi}$ demonstrated by fluorescent pixel counting (Fig 2C and 2D). These results clearly indicate that EPT protects zebrafish from M. abscessus infection.

\section{EPT targets LeuRS in M. abscessus}

Benzoxaboroles were shown to inhibit LeuRS in fungi, gram-negative pathogens and most recently M. tuberculosis $[11,19,28]$. To determine the target of EPT in M. abscessus, $10^{9}$ CFUs were plated on solid media with 10X, 20X, or 40X MIC 90 of EPT to select EPT-resistant mutants. Control mutants were also selected on 40X MIC of AMK. Unlike the EPT resistance frequency of $4.8 \times 10^{-8}$ in Pseudomonas aeruginosa, the resistance frequency was 10-fold lower in M. abscessus (S4 Table, $2 \times 10^{-9}$ ) [28]. The low in vitro resistance frequency to EPT is highlighted when compared to the control mutants against $\mathrm{AMK}\left(1.3 \times 10^{-8}\right)$. In order to confirm that isolated resistant mutants were true mutants to EPT, we performed REMA using a panel of antimycobacterial agents on the four mutants (one selected on 10X, one on 20X, and two on $40 \mathrm{X} \mathrm{MIC} \mathrm{M}_{90}$ of EPT). We also screened one AMK mutant. As illustrated in S3 Fig, the four EPT mutants were resistant up to $2.7 \mu \mathrm{g} / \mathrm{mL}$ EPT, while maintaining susceptibility to AMK, bedaquiline (BDQ), and RIF. Likewise, the AMK control mutant was only resistant to AMK.

Since benzoxaboroles are known to target LeuRSs, we used a focused approach to identify the mutation(s) in leuS that could be responsible for the observed EPT resistance. leuS was PCR amplified from gDNA from the four EPT mutants, and single-nucleotide polymorphisms (SNPs) were identified using Sanger sequencing (S5 Table). In all four mutants, a G to C transversion at position 1306 was identified, which resulted in the conserved $\mathrm{D} 436$ residue critical for the catalysis of editing misaminoacylated tRNA ${ }^{\text {Leu }}$ substituted for H436 (Fig 3A and 3B) [29]. This was further supported with whole-genome sequencing of the M. abscessus ATCC 19977 reference strain, one mutant isolated at 20X MIC, and one mutant isolated at 40X MIC. When compared to the reference strain, five variants were identified in the 20X mutant including a $\mathrm{T}$ to $\mathrm{G}$ transition at position $1261 \mathrm{in}$ leuS which resulted in a Y421D substitution. Furthermore, the $\mathrm{G}$ to $\mathrm{C}$ transversion at position 1306 which lead to the $\mathrm{D} 436 \mathrm{H}$ substitution was confirmed in the 40X mutant ( $\$ 6$ Table). This contrasts with LeuRS variants identified in $P$. aeruginosa such as T323P, T327P, and V429M from Hernandez et al. or LeuRS variants such as T322I, D326N, A414V, and R435H identified in a phase 2 clinical trial for complicated urinary tract infections caused by E. coli from O'Dwyer et al. [28,30]. However, the phase 2 clinical trial did identify E. coli isolates with a LeuRS D436A variant. (M. abscessus numbering). To verify the functional significance of the $\mathrm{D} 436 \mathrm{H}$ substitution, the leuS genes from M. abscessus ATCC 19977 wildtype (EPT sensitive) and M. abscessus containing the G1306C substitution (EPT-resistant) were cloned into the mycobacterial integrative vector pMV306 under the con-

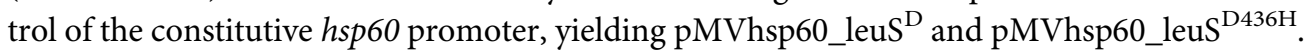
EPT-sensitive M. abscessus was electroporated with pMVhsp60_leuS ${ }^{\mathrm{D}}$, pMVhsp60_leuS ${ }^{\mathrm{D} 436 \mathrm{H} \text {, }}$ or empty vector control. Only EPT sensitive M. abscessus complemented with the mutant leuS (pMVhsp60_leuS ${ }^{\mathrm{D} 436 \mathrm{H}}$ ) and not the wildtype leuS (pMVhsp60_leuS ${ }^{\mathrm{D}}$ ) had increased resistance to EPT (Fig 3C). The integrative complement system using pMVhsp60 may not have 
A

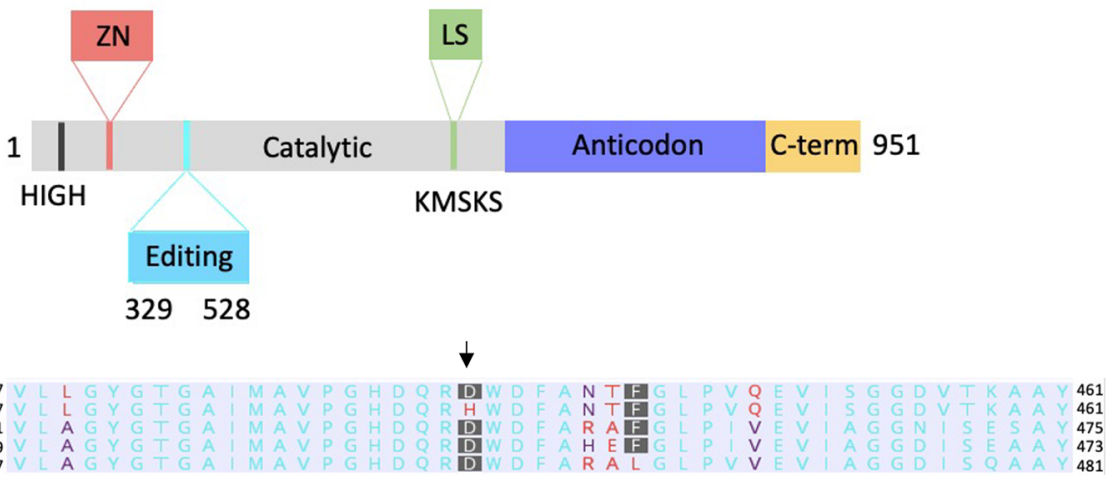

C

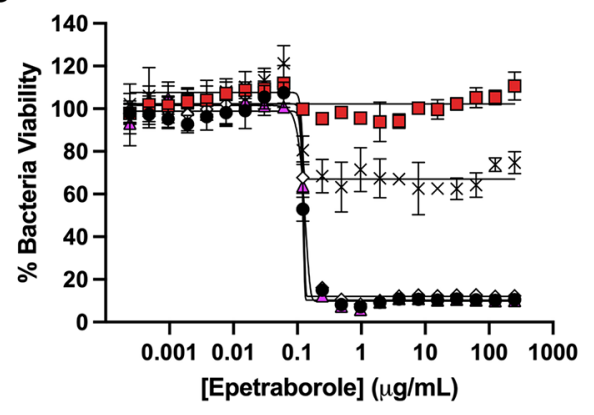

E

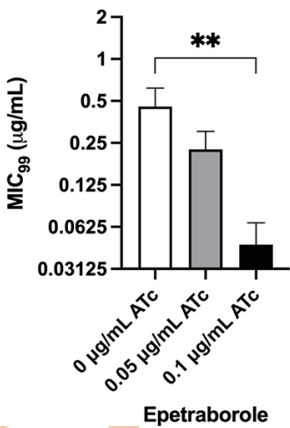

D

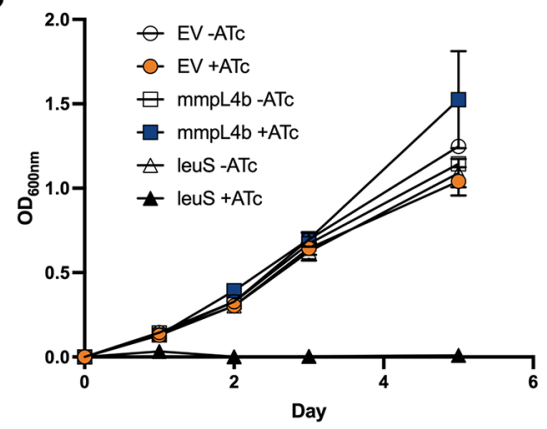

Fig 3. EPT targets the editing domain of LeuRS in M. abscessus. A Domains of M. abscessus LeuRS. Zinc domain, pink; editing domain, cyan; catalytic domain, grey; leucine-rich domain, green; anticodon-binding domain, purple; cterminus, yellow. B Amino acid alignment of part of the editing domain of LeuRS M. abscessus ATCC 19977, EPT mutant, and reference mycobacteria. Non-consensus residues are in red. Genbank accession numbers: P9WFV1.1 ( $M$. tuberculosis) 11896261 (M. avium) 6224275 (M. marinum). C Dose-response curves of M. abscessus ATCC 19977 (black circles), D436H mutant (red squares), M. abscessus empty vector (white diamonds), M. abscessus pMVhsp60_leuS (pink triangles) and M. abscessus pMV306hsp60_leuSD436H (crosses). Data shown is mean \pm SD from one experiment representative of two. $\mathbf{D}$ leuS is an essential gene in M. abscessus using CRISPRi gene knockdown. M. abscessus carrying integrated empty CRISPRi vector (EV, circles), CRISPRi: $m m p L 4 b$ as a non-essential gene control (squares), or CRISPRi:leuS (triangles) were grown with/without $0.01 \mu \mathrm{g} / \mathrm{mL} \mathrm{ATc}$. Data is mean \pm SD from biological triplicates. E MIC ${ }_{99}$ to EPT and RFB in CRISPRi knockdown of leuS in M. abscessus. ${ }^{* *}$, p $=0.005$; ns, no statistical significance by one-way ANOVA with Dunnett's multiple comparisons test. Data shown is mean \pm SD from three biological replicates.

https://doi.org/10.1371/journal.ppat.1009965.g003 
provided a complete phenocopy of the true mutant when compared to the episomal equivalent. Also, M. abscessus may not use the $h s p 60$ promoter from M. bovis with optimal efficiency. Lastly, the $h s p 60$ promoter may not operate with the same strength as the leuS promoter.

We showed that LeuRS ${ }^{\text {D436H }}$ confers high-level resistance to EPT and that complementing the resistant allele into a wildtype background imparts resistance. As a complementary means of verifying the target, we adapted a CRISPR-interference (CRISPRi) system for gene knockdown in M. abscessus [31]. CRISPRi facilitates gene knockdown using a guide RNA and catalytically inactive Cas9 endonuclease to sterically prevent transcription of a gene of interest when induced with anhydrotetracycline (ATc). We used CRISPRi to knockdown leuS which resulted in hyper susceptibility to EPT. Since leuS is an essential gene in M. abscessus (Fig 3D), we used 10-fold and 20-fold less ATc than required for complete growth suppression when targeting an essential gene. The EPT MIC 99 of the strain induced with $0.1 \mu \mathrm{g} / \mathrm{mL}$ ATc decreased 10-fold compared to uninduced conditions, while induction did not affect the rifabutin (RFB) $\mathrm{MIC}_{99}$ (Fig 3E). These results provide further evidence that EPT targets LeuRS in M. abscessus.

\section{EPT binds the editing active site of LeuRS}

Although EPT is active against M. abscessus and M. tuberculosis, the $\mathrm{MIC}_{90}$ for M. abscessus whole-cell activity is 7 -fold lower ( $\mathrm{MIC}_{90 \mathrm{Mabs}} 0.063 \mu \mathrm{g} / \mathrm{mL}$ vs $\mathrm{MIC}_{90 \mathrm{Mtb}} 0.46 \mu \mathrm{g} / \mathrm{mL}$ ). We hypothesized that EPT had a higher affinity for the M. abscessus LeuRS editing active site. We therefore performed binding studies between EPT and the editing domains of LeuRS from $M$. abscessus and M. tuberculosis using isothermal titration calorimetry (ITC) (S4 Fig). M. abscessus LeuRS and M. tuberculosis LeuRS contain a single binding site for EPT, and we obtained similar equilibrium dissociation constants and Gibbs free energies (Table 2). The EPT $-M$. abscessus LeuRS binding has a higher enthalpic contribution (1.1 to $\left.3.2 \mathrm{kcal} \mathrm{mol}^{-1}\right)$, while the EPT-M. abscessus LeuRS binding has a lower entropic contribution (5.5 to $3.3 \mathrm{kcal} \mathrm{mol}^{-1}$ ).

To gain insight into the interactions between EPT and LeuRS, we solved the crystal structure of the M. abscessus editing domain (LeuRS ${ }_{303-498}$ ). We obtained the crystal structures of the LeuRS editing domain unliganded 2.1 A resolution (PDB 7N11) and in complex with the adenosine monophosphate (AMP) adduct with EPT at $1.7 \AA$ resolution (PDB 7N12, S7 Table). AMP acts as a surrogate for the 3 ' end of the tRNA acceptor stem. In concordance with the binding mode of action of benzoxaboroles [19], we detected strong electron density in the

Table 2. Thermodynamic analysis of EPT binding with LeuRS.

\begin{tabular}{l|l|l|l|l}
\hline Bacteria & $\Delta \mathbf{G}\left(\mathbf{k c a l ~ m o l}^{-\mathbf{1}}\right)$ & $\mathbf{\Delta H}\left(\mathbf{k c a l ~ m o l}^{-\mathbf{1}}\right)$ & $-\mathbf{T} \Delta \mathbf{S}\left(\mathbf{k c a l ~} \mathbf{~ m o l}^{-\mathbf{1}}\right)$ & $\mathbf{K d ~}(\boldsymbol{\mu M})$ \\
\hline M. abscessus & $-6.49 \pm 0.07$ & $-3.2 \pm 0.4$ & $-3.3 \pm 0.3$ & $16 \pm 4$ \\
\hline M. tuberculosis & $-6.9 \pm 0.2$ & $-1.1 \pm 0.3$ & $-5.5 \pm 0.5$ & $10 \pm 4$ \\
\hline
\end{tabular}

$\Delta \mathrm{G}$, change in Gibbs free energy; $\Delta \mathrm{H}$, change in enthalpy; $\Delta \mathrm{S}$, change in entropy; $T$, temperature (303K)

$\mathrm{Kd}$, dissociation constant

https://doi.org/10.1371/journal.ppat.1009965.t002 
active site corresponding to the EPT-AMP adduct formed through covalent interactions between the boron atom of EPT and the 2' and 3' hydroxyl groups on the ribose ring of AMP (Figs 4A and S5A). Comparing unliganded and liganded residues in M. abscessus editing domain structures shows a sizable shift of residues 416-422, located around the adenosine pocket, upon drug binding, including ordering of Y421 to interact with the EPT-AMP adduct phosphate, and a decrease of B factors in the neighbouring residues (S5B Fig). This increase in order could explain the relatively lower entropic contribution to binding observed in ITC, although an unliganded $M$. tuberculosis structure is not available for comparison.

Once bound with benzoxaborole-AMP adduct, the active sites of $M$. tuberculosis (PDB: 5AGR) [19] and M. abscessus LeuRS, and mode of adduct binding are very similar (Figs 4A and $55 \mathrm{C} 2$ ). Both proteins make critical contacts with the primary amine side chain through D433 (D447 in M. tuberculosis) and the universally conserved D436 (D450 in M. tuberculosis) (Fig 4A). In addition, R435 (R449 in M. tuberculosis) hydrogen bonds with the ethoxy oxygen of EPT and packed with the ribose of AMP (Fig 4A). The packing interaction on the ribose of AMP is underscored from E. coli mutants with $\mathrm{R} 435 \mathrm{H}$ variants that lead to resistance early in the phase 2 clinical trial for complicated urinary tract infections. EPT has a hydroxypropyl ether moiety, rather than the ethyl ether in the benzoxaborole bound to M. tuberculosis LeuRS, but the extension shows very weak electron density and likely does not contribute to binding. The only notable difference is a $\sim 1$ shift in phosphate group of the EPT-AMP adduct (Fig 4B). In both complexes, the phosphate is pinned between Y421 (Y435) and T323 (T337). The electron density indicates that there are multiple rotomeric conformations of T323, meaning its hydroxyl could hydrogen bond with the phosphate or with the 3' $\mathrm{O}$ and ether oxygen of the adduct (Fig 4B). M. tuberculosis LeuRS T337 is modelled in the latter position, but inspection of deposited maps also indicates multiple rotomeric conformations. Variants at T322, T323 and T327 were identified in P. aeruginosa and E. coli mutants resistant to benzoxaboroles, which supports the importance of the threonine rich region for editing activity [32]. From the structural data, we propose that EPT binds LeuRS from M. abscessus and M. tuberculosis in a similar manner with similar affinity, providing a shared pathway for future structure-activity relationship analysis.

\section{Norvaline is toxic to editing-deficient EPT mutants}

Since resistance to EPT was shown in a phase 2 clinical trial of complicated urinary tract infections from E. coli, we sought a way to minimize the emergence of resistance in M. abscessus pulmonary infections [30]. We hypothesized that norvaline, a non-proteinogenic amino acid absent from extant proteins [33], would be toxic to M. abscessus editing-deficient mutants that acquired EPT resistance. When challenged with $59 \mu \mathrm{g} / \mathrm{mL}$ norvaline, the D436H editing-deficient mutant was significantly reduced in growth, while the wild-type strain was not inhibited (Fig 5A). The mutant strain complemented with wild-type leuS (D436H::leuS ${ }^{\mathrm{D}}$ ) displayed a two-day lag before significant growth. As controls, leucine and isoleucine were not toxic to the $\mathrm{D} 436 \mathrm{H}$ mutant. There was however a minor reduction in mutant growth in $59 \mu \mathrm{g} / \mathrm{mL}$ valine, but no growth difference between the $\mathrm{D} 436 \mathrm{H}$ and $\mathrm{D} 436 \mathrm{H}:$ :leuS ${ }^{\mathrm{D}}$ strains. Thus, we cannot conclude that valine exerts some toxicity to editing-deficient strains. Next, we asked if norvaline toxicity could be rescued with branched-chain amino acids (BCAAs). The D436H mutant was grown in $59 \mu \mathrm{g} / \mathrm{mL}$ norvaline with varying concentrations of BCAAs. Leucine but not isoleucine or valine rescued growth (Fig 5B). These observations corroborate the results that LeuRSs contain natural selectivity with their aminoacylation site for leucine and can discriminate against isoleucine and valine, but to a lesser extent against norvaline as non-cognate amino acids [34]. 
A

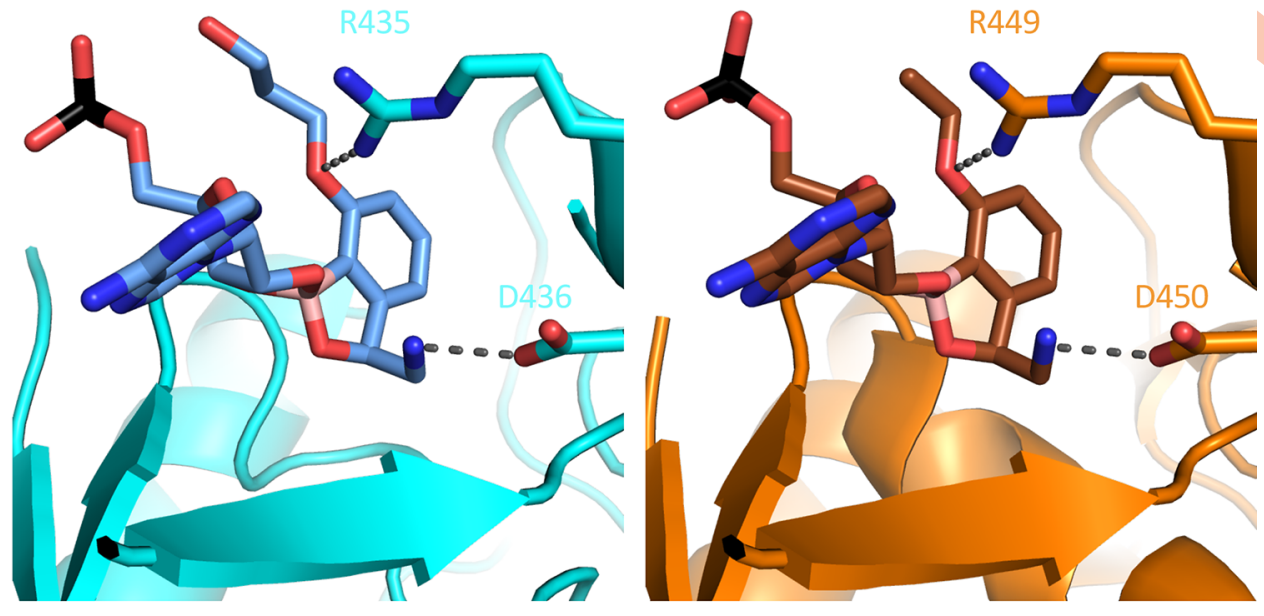

B
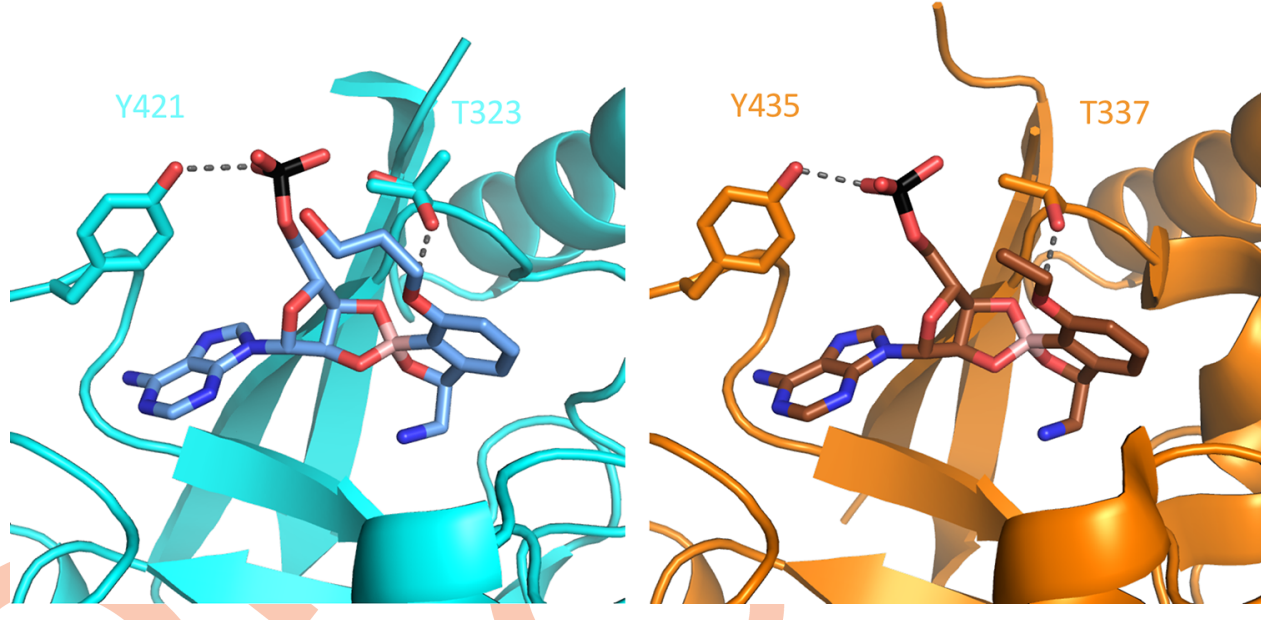

Fig 4. Similar binding of benzoxaboroles to the editing domain of LeuRS. EPT-AMP (blue) bound in the active site of M. abscessus LeuRS (cyan) and a similar benzoxaborole-AMP (brown) bound in active site of M. tuberculosis LeuRS (PDB 5AGR) (orange). A Critical arginine and aspartic acid residues make similar interactions with benzoxaboroles. B Phosphate from AMP pivots towards threonine residue in M. abscessus LeuRS. Conformer 1 of T323 points OH towards EPT, while conformer 2 orients $\mathrm{OH}$ towards the phosphate.

https://doi.org/10.1371/journal.ppat.1009965.g004

\section{Norvalination of the proteome induces the heat shock response}

To provide a mechanism of norvaline toxicity, we analyzed the proteomes of wild-type,

$\mathrm{D} 436 \mathrm{H}$ mutant, and $\mathrm{D} 436 \mathrm{H}: \mathrm{leuS}^{\mathrm{D}}$ grown in norvaline or valine as control. We analyzed $\sim 2500$ proteins from whole-cell lysates after 12 hours or 3 days of incubation in $59 \mu \mathrm{g} / \mathrm{mL}$ norvaline or valine using reverse-phase HPLC/MS. Proteins filtered for leucine residues $14 \mathrm{Da}$ lighter (14 Da corresponds to missing methylene group on norvaline) were enumerated using spectral counting. The wild-type maintained preferential incorporation of leucine into proteins, while the $\mathrm{D} 436 \mathrm{H}$ mutant had a median norvaline misincorporation in $6 \%$ of the proteome after 12 hours. However, the amount of norvaline misincorporation between the $\mathrm{D} 436 \mathrm{H}$ mutant and the $\mathrm{D} 436 \mathrm{H}::$ leuS ${ }^{\mathrm{D}}$ strain was not significantly different (Fig 6A). Given that there was a twoday delay when the complemented strain was grown in norvaline (Fig 5A), we hypothesized 
A
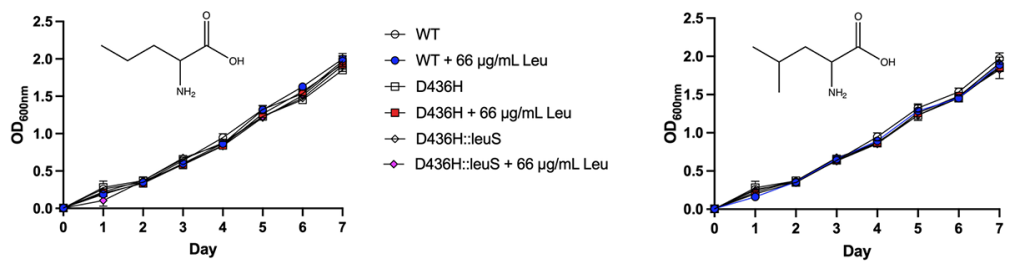

- $W T$

- $W T+66 \mu \mathrm{g} / \mathrm{mL}$ lle

$\rightarrow \mathrm{D} 436 \mathrm{H}$

- $\mathrm{D} 436 \mathrm{H}+66 \mu \mathrm{g} / \mathrm{mL}$ lle

$\rightarrow$ D436H::leus

- D D436H:IeuS + $66 \mu \mathrm{g} / \mathrm{mL}$ lle

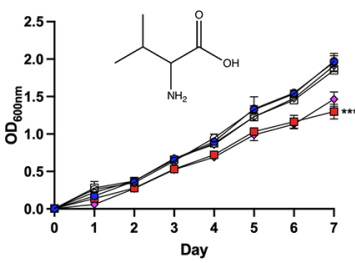

$-W T$

- $W T+59 \mu \mathrm{g} / \mathrm{mL}$ Val

— $\mathrm{D} 436 \mathrm{H}$

$\rightarrow \mathrm{D} 436 \mathrm{H}+59 \mu \mathrm{g} / \mathrm{mL}$ Val

$\rightarrow$ D436H::leus

$\rightarrow$ D $436 \mathrm{H}:: \mathrm{leuS}+59 \mu \mathrm{g} / \mathrm{mL}$ Val

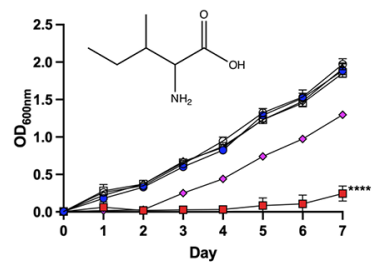

$-w T$

- $W T+59 \mu \mathrm{g} / \mathrm{mL}$ Nva

— $\mathrm{D} 436 \mathrm{H}$

$\rightarrow-D 436 \mathrm{H}+59 \mu \mathrm{gg} / \mathrm{mL}$ Nva

$\rightarrow \mathrm{D} 436 \mathrm{H}: \mathrm{lleuS}$

$\rightarrow$ D $436 \mathrm{H}: \mathrm{leuS}+59 \mu \mathrm{g} / \mathrm{mL} \mathrm{Nva}$

B
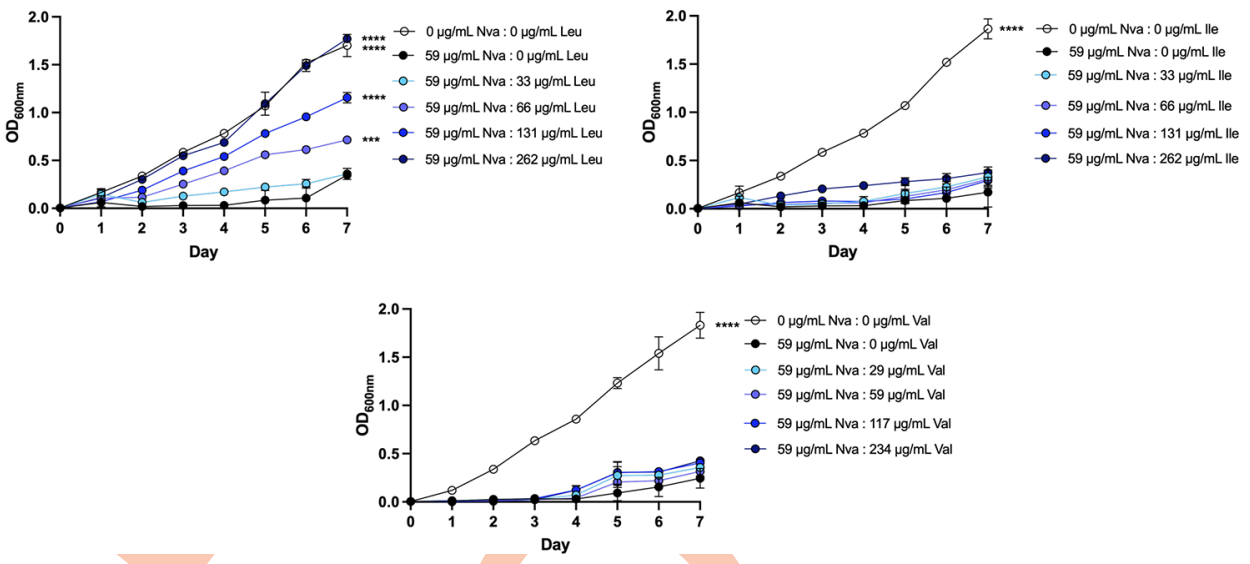

Fig 5. Norvaline is toxic to M. abscessus D436H editing deficient mutants. A ATCC 19977, D436H mutant, and D436H:leuS ${ }^{\mathrm{D}}$ complement strains were grown in Sauton media with $59 \mu \mathrm{g} / \mathrm{mL}$ norvaline (top left), $66 \mu \mathrm{g} / \mathrm{mL}$ leucine (top right), $59 \mu \mathrm{g} / \mathrm{mL}$ valine (lower left), or $66 \mu \mathrm{g} / \mathrm{mL}$ isoleucine (lower right). Data shown is from three biological replicates, one-way ANOVA with Tukey's multiple comparisons test. ${ }^{* * *}, \mathrm{p}<0.0001$. B D436H mutant grown in Sauton media with $59 \mu \mathrm{g} / \mathrm{mL}$ norvaline and a range of leucine (left), isoleucine (middle), or valine (right). D436H mutant grown in $0 \mu \mathrm{g} / \mathrm{mL}$ norvaline and $0 \mu \mathrm{g} / \mathrm{mL}$ BCAA represents growth control. D436H mutant grown in $59 \mu \mathrm{g} /$ $\mathrm{mL}$ norvaline and $0 \mu \mathrm{g} / \mathrm{mL}$ BCAA represents inhibited growth control. BCAAs added to cultures from $33 \mu \mathrm{g} / \mathrm{mL}$ to $262 \mu \mathrm{g} / \mathrm{mL}$ (Leu/Ile) or $29 \mu \mathrm{g} / \mathrm{mL}$ to $234 \mu \mathrm{g} / \mathrm{mL}$ (Val). Data shown is mean \pm SD from three biological replicates. Groups compared to inhibited growth control with one-way ANOVA with Dunnett's multiple comparisons test. ${ }^{* * *}$, $\mathrm{p}=0.0009 ;{ }^{* * * *}, \mathrm{p} \leq 0.0001$

https://doi.org/10.1371/journal.ppat.1009965.g005

that the effects of gene complementation would be seen at a later time point when grown in $59 \mu \mathrm{g} / \mathrm{mL}$ norvaline. The experiments were repeated, and the strains were incubated for 3 days. Again, the median norvalination increased in the $\mathrm{D} 436 \mathrm{H}$ mutant relative to wild-type, but the level of misincorporated norvaline increased to $20 \%$ of the proteome. At this time point, the complemented strain partially rescued the level of norvalination after 3 days (Fig $6 \mathrm{~B})$. In all conditions tested, we measured background misaminoacylation of valine in the proteome (Fig 6A and 6B).

Next, we asked how the cell responded to stress caused by norvaline misincorporation in the proteome. Using total spectral counting, we determined the fold change in protein abundance from $\mathrm{D} 436 \mathrm{H}$ mutant relative to wild-type. There was a distinct increase in proteins 
A

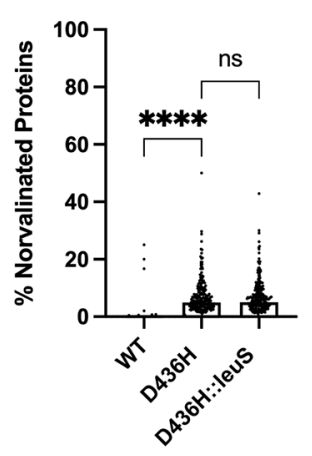

C

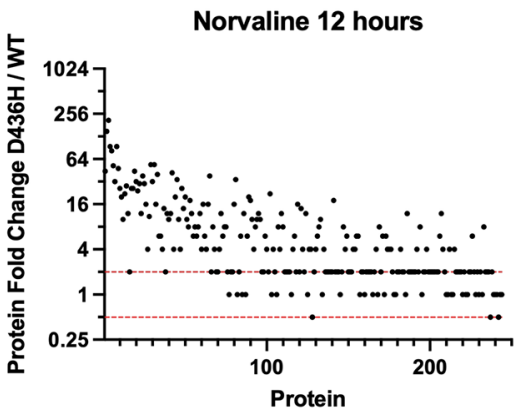

$\mathbf{E}$

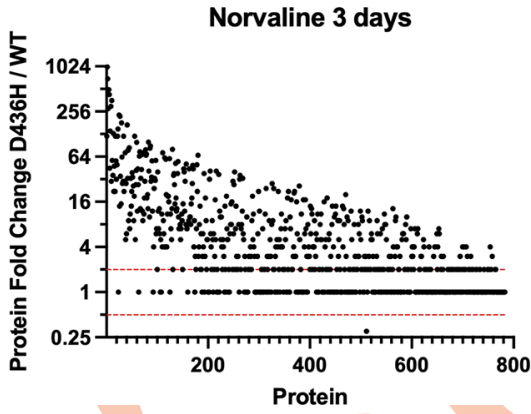

B
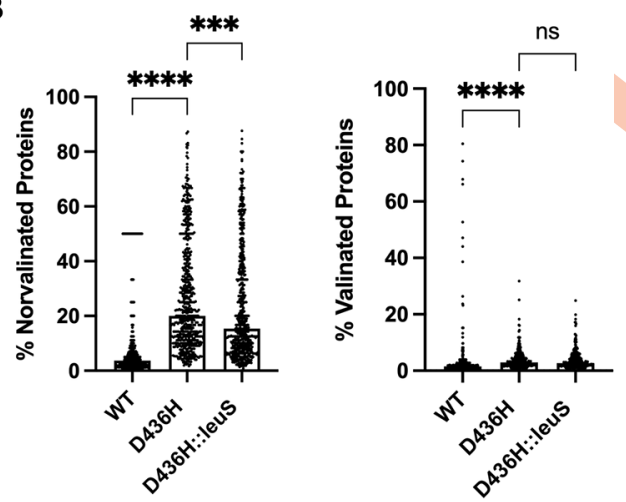

D

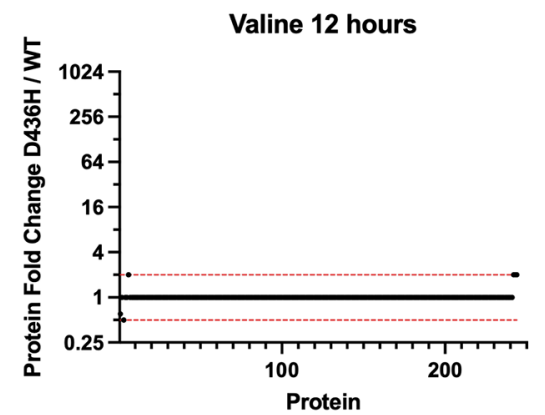

$\mathbf{F}$

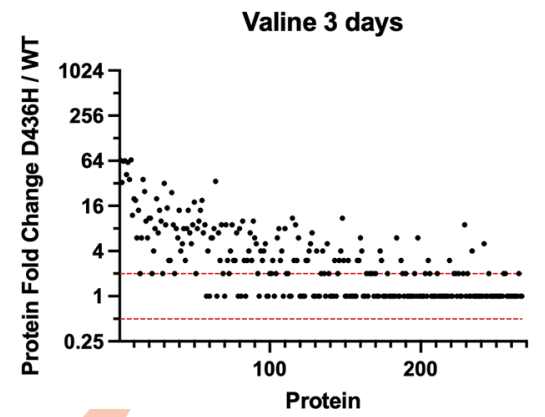

Fig 6. Norvaline misaminoacylation in editing deficient mutants alters the proteomic landscape. A-B $M$. abscessus ATCC 19977 or D436H mutant was grown in $59 \mu \mathrm{g} / \mathrm{mL}$ valine or $59 \mu \mathrm{g} / \mathrm{mL}$ norvaline for 12 hours or 3 days. Valine was used as a specificity control. Total cell lysate was collected. (Nor)valinated protein medians from the proteomes were compared using Kruskal-Wallis with Dunn's multiple comparisons test. ${ }^{* * *}, \mathrm{p}=0.0005$; ${ }^{* * * *}$, $\mathrm{p}<0.0001$; ns, no statistical significance. C-F Protein fold abundance shown as D436H mutant relative to M. abscessus ATCC 19977. Dashed red lines indicate $0.5 \mathrm{x}$ and $2 \mathrm{x}$ fold abundance thresholds for biologically relevant changes.

https://doi.org/10.1371/journal.ppat.1009965.g006

when challenged with norvaline that was absent from the valine control (Fig 6C-F). We used the protein-protein interaction (PPI) mapping tool STRING to identify upregulated proteins with common functions. When 130 of the most highly abundant proteins after norvaline stress were mapped for PPIs (Fig 7A), proteins belonging to the Clp protease family (ClpP1, ClpP2, $\mathrm{ClpX}$ ) and GroEL chaperonin family (GroEL, GroL2, GroS) were identified (False discovery rate $7.2 \times 10^{-4}$ at $12 \mathrm{~h}, 2.1 \times 10^{-5}$ at $3 \mathrm{~d}$ ) (Fig $7 \mathrm{C}$ ). As a control, 130 randomly selected proteins from the norvaline dataset were not enriched for chaperonins and proteases when analyzed for PPIs (Fig 7B). We also observed a temporal change in chaperonin and protease levels where early stress from norvaline at 12 hours upregulated chaperonins followed by protease 
A

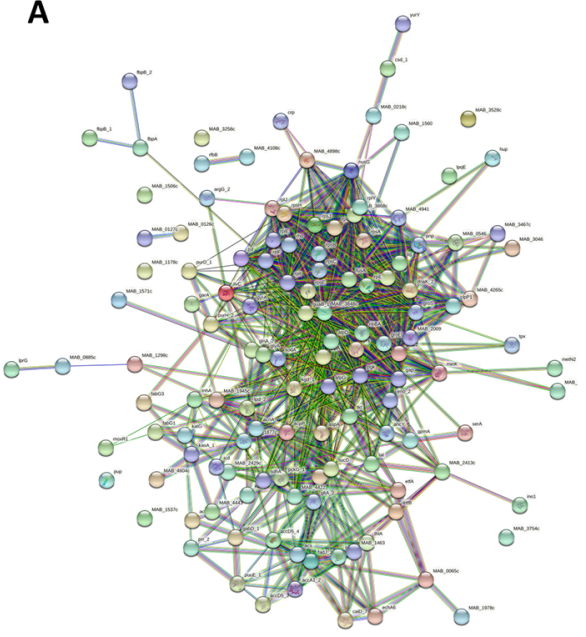

C

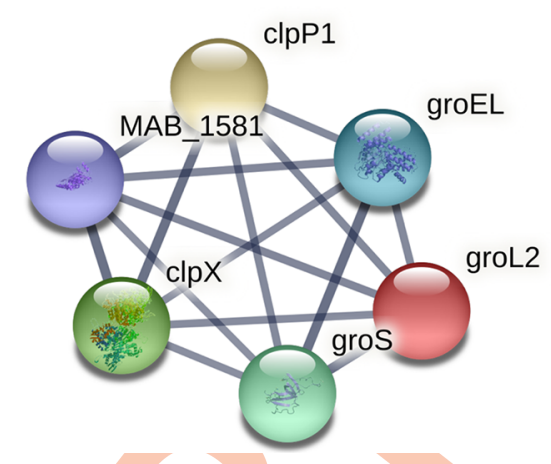

B

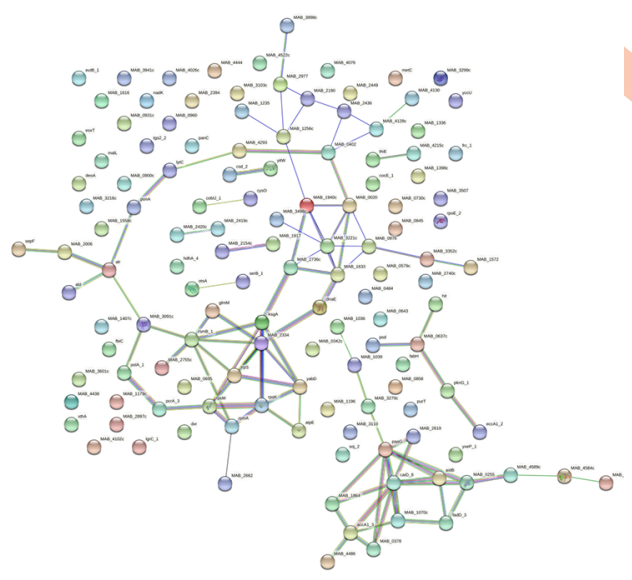

D

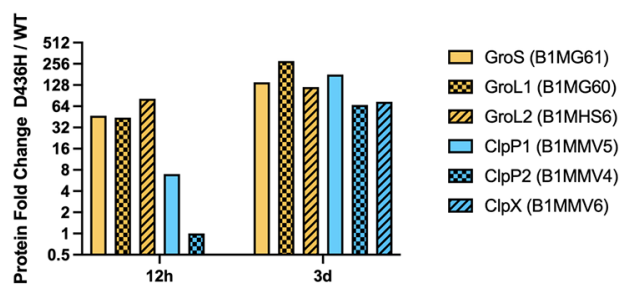
Fig 7. Norvaline stress induces the heat shock response in editing deficient mutants. A Protein-protein interaction
network from the 130 most abundant proteins in $\mathrm{D} 436 \mathrm{H}$ mutant when challenged with $59 \mu \mathrm{g} / \mathrm{mL}$ norvaline for 12 hours. 942 PPIs identified with PPI enrichment $\mathrm{p}$-value $<1.0 \times 10^{-16}$. B PPI network from 130 randomly selected proteins in D436H mutant when challenged with $59 \mu \mathrm{g} / \mathrm{mL}$ norvaline for 12 hours. 102 PPIs identified with PPI enrichment p-value of 0.0397. C Clp protease/chaperonin family local network cluster identified in a (false discovery rate: 0.00072 ). D Temporal signature of the heat shock response. Protein expression levels of D436H norvaline relative to WT norvaline were measured by spectral counting.

https://doi.org/10.1371/journal.ppat.1009965.g007

upregulation at 3 days (Fig 7D). This data suggests that norvaline misincorporation into the proteome results in toxicity from misfolded proteins $[14,33,35-40]$.

\section{Norvaline reduces resistance to EPT in vitro and potentiates EPT activity in vivo}

Knowing that norvaline targets LeuRS editing domain, we asked if norvaline could prevent escape mutants to EPT in vitro. We observed a 23 -fold reduction in escape mutant frequency when M. abscessus ATCC 19977 was plated on 7H10 plates containing 10X MIC ${ }_{90}$ EPT and $590 \mu \mathrm{g} / \mathrm{mL}$ norvaline $\left(1.89 \times 10^{-10}\right)$ over EPT alone $\left(4.28 \times 10^{-9}\right)$ (Fig 8A); as a control, RFB did not benefit from norvaline supplementation. To test whether this effect would be observed in other mycobacteria, we repeated with $M$. tuberculosis, again observing a suppression of EPT mutants with norvaline exposure (Fig 8A). Next, the $\mathrm{MIC}_{90}$ of EPT against M. abscessus ATCC 19977 was measured with differing doses of norvaline (Fig 8B). Norvaline had no effect on the $\mathrm{MIC}_{90}$ even up to $590 \mu \mathrm{g} / \mathrm{mL}$. These results suggest norvaline does not act as a traditional adjuvant to EPT. 
A

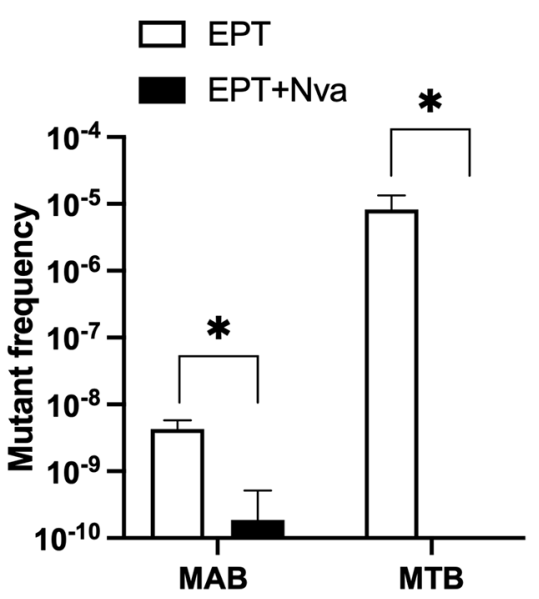

B

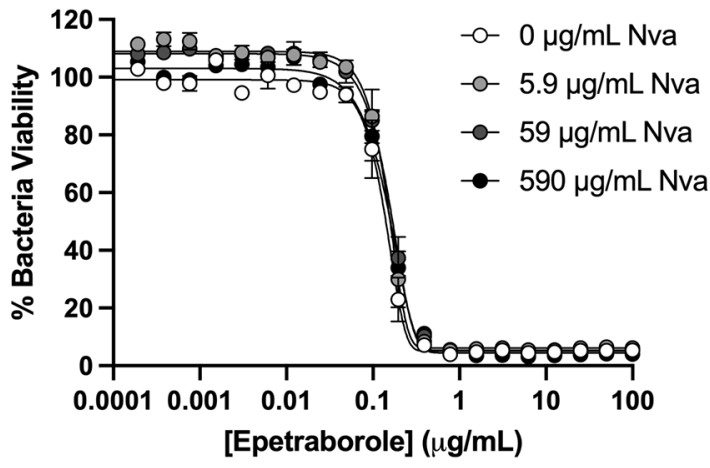

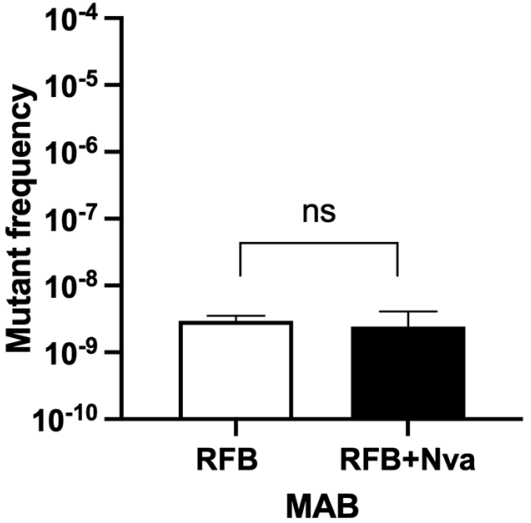

C

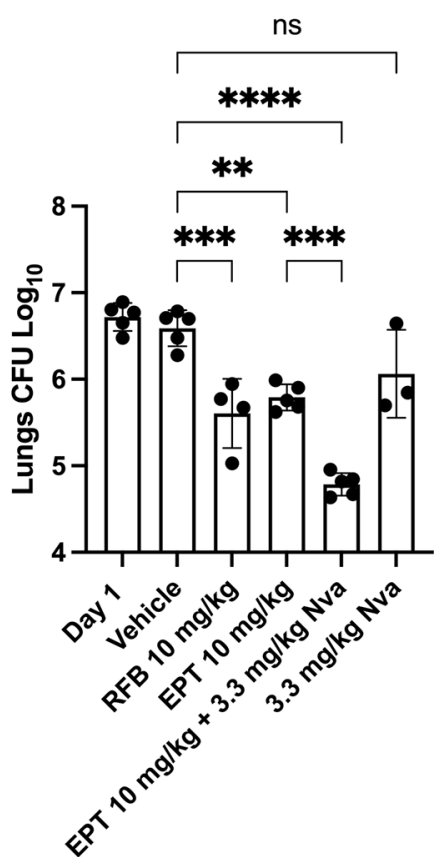

Fig 8. Norvaline improves epetraborole efficacy in vitro and in vivo. A Mutant frequency to $10 \mathrm{X} \mathrm{MIC} \mathrm{C}_{90} \mathrm{EPT}$ $(0.63 \mu \mathrm{g} / \mathrm{mL})$ or $10 \mathrm{X} \mathrm{MIC}_{90}$ EPT $+10 \mathrm{X} \mathrm{MIC}_{90}$ norvaline $(590 \mu \mathrm{g} / \mathrm{mL})$ in M. abscessus ATCC 19977 and M. tuberculosis H37Rv. Data shown is mean \pm SD from biological triplicates. Means compared using Poisson distribution. *, $\mathrm{p}<0.0001$; ns, no statistical significance. B $M$. abscessus susceptibility to EPT with norvaline as an adjuvant. Data shown is mean \pm SD from one experiment representative of three. C EPT in vivo activity in SCID mouse model of $M$. abscessus lung infection with norvaline supplementation. Rifabutin (RFB) acts as positive control. Data shown is mean \pm SD of three to five mice per treatment group. Means compared using one-way ANOVA with Tukey's multiple comparisons test. ${ }^{* *}, \mathrm{p}<0.007 ;{ }^{* * *}, \mathrm{p}<0.0005 ;{ }^{* * * *} ; \mathrm{p}<0.0001 ; \mathrm{ns}$, no statistical significance.

https://doi.org/10.1371/journal.ppat.1009965.g008

We evaluated EPT and norvaline combination therapy for in vivo activity in a NOD.SCID mouse model of M. abscessus infection [41]. Mice were infected intranasally with a high inoculum of $M$. abscessus ( $\left.10^{6} \mathrm{CFU}\right)$. Treatment was started 1-day post-infection with once-daily oral vehicle (carboxymethylcellulose), RFB as positive control (10 mg/kg), EPT (10 mg/kg), $\mathrm{EPT}+$ norvaline $(10 \mathrm{mg} / \mathrm{kg}+3.3 \mathrm{mg} / \mathrm{kg})$, or norvaline $(3.3 \mathrm{mg} / \mathrm{kg})$. Norvaline was dosed at 3.3 
$\mathrm{mg} / \mathrm{kg}(5 \mathrm{mM})$ which represents $\sim 10 \mathrm{X} \mathrm{MIC}_{90}$ in vitro against the editing deficient $\mathrm{D} 436 \mathrm{H}$ mutant. Previously, norvaline had been shown to be an effective in vivo neuroprotective agent as an arginase inhibitor at $2 \mathrm{mM}$ in a triple-transgenic mouse model of Alzheimer's disease [42]. Compared to vehicle-treated mice, we measured a $1 \log _{10}$ decrease in bacterial burden in the lungs from RFB or EPT treatment. Furthermore, the addition of norvaline to the EPT group was significantly more active than EPT alone with an additional $\sim 1 \log _{10}$ decrease in bacterial burden in the lungs while norvaline alone had no significant effect (Fig $8 \mathrm{C}$ ).

\section{Discussion}

Our data and two recent publications $[43,44]$ confirmed the activity of benzoxaboroles against mycobacteria using zebrafish and murine models of infection and we showed that benzoxaboroles can be potentiated when combined with norvaline. Specifically, we demonstrated that the combination of EPT with norvaline reduces the emergence of M. abscessus and M. tuberculosis mutants and results in increased activity in vivo compared to EPT. Whether norvaline can be a therapeutic adjunct for other benzoxaboroles remains to be established, but is supported by observations with tavaborole and norvaline in E. coli [45]. The potential for combination therapy may be of value in non-mycobacterial applications, such as the treatment of urinary tract infections, where EPT did not progress beyond phase 2 studies owing to the rapid emergence of resistance [30].

While we documented that EPT is active against both M. abscessus and M. tuberculosis in vitro, unexpectedly, we observed that EPT is more active against $M$. abscessus, which conflicts with the standard experience in antimycobacterial drug discovery [6,46-51]. Our cocrystal structure and ITC data point to similarities and differences in the interaction of EPT with the respective LeuRS proteins. Most notably, while the Kd values were similar, the enthalpy was greater for the EPT interaction with LeuRS of M. abscessus. Knowing that compounds with more enthalpically favourable binding are prioritized in hit-to-lead optimization [52], it may be possible that the discordance in enthalpy in part explains the difference in biological activity of EPT against the two organisms (MIC $90 \mathrm{Mabs} 0.063 \mu \mathrm{g} / \mathrm{mL}$ vs $\mathrm{MIC}_{90 \mathrm{Mtb}} 0.46 \mu \mathrm{g} / \mathrm{mL}$ ). Other possibilities explaining the difference in whole-cell activity may be linked to differential expression level of leuS in both species, the lack of efflux pumps in M. abscessus, or the inability of M. abscessus to survive conditions that mimic leucine starvation, although these remain to be addressed. At a minimum, the current data can be used for future structure-activity relationship studies aimed at uncovering analogues with improved potency against LeuRS of $M$. abscessus, M. tuberculosis and other mycobacteria.

Our in vivo data shows the value of EPT alone in rescuing zebrafish embryos from lethal $M$. abscessus infection and that EPT + norvaline can control experimental pulmonary M. abscessus infection to a greater degree than the control antibiotic, RFB. Rather than acting as a traditional adjuvant like $\beta$-lactamase inhibitors for $\beta$-lactams [53], we propose that norvaline potentiates EPT in vitro activity by maintaining a pharmacological pressure on M. abscessus to remain editing proficient and limit the toxicity induced by the unfolded protein response and thus, limiting escape mutants. However, given that our in vivo model of pulmonary M. abscessus infection in NOD.SCID mice does not exceed a bacterial burden of $\sim 10^{7}$ in the lungs and that the rate of resistance against EPT is $\sim 10^{-9}$, other mechanisms might better explain our findings. Since the NOD.SCID model is immunocompromised for NK cells and adaptive immunity, macrophages-the most notable front-line defence for mycobacterial pulmonary infections-would be the main immune cell responsible for controlling the M. abscessus infection. One of the primary mechanisms of macrophages for controlling infections is the production of reactive nitrogen species like nitric oxide $(\mathrm{NO})$ from inducible nitric oxide synthase (iNOS). Because NO is produced from arginine by iNOS, macrophages must regulate arginine 
metabolism between iNOS and the urea cycle, which naturally metabolizes arginine through the enzyme arginase. Interestingly, norvaline was shown to be an inhibitor of arginase and this resulted in increased production of NO in activated macrophages [54,55]. This increased production of NO from arginase inhibition might explain the lower bacterial burden observed in the NOD.SCID mouse group that received EPT + norvaline treatment.

Given that response rates to M. abscessus treatment are poor, resulting in chronic and potentially untreatable pulmonary and dermatologic infections, we submit that benzoxaboroles with the addition of a leucine mimic hold promise for the treatment of $M$. abscessus infections, and potentially other mycobacterial infections, such as tuberculosis.

\section{Materials and methods}

\section{Ethics statement}

All protocols involving mice followed the guidelines of the Canadian Council on Animal Care (CCAC) and were approved by the ethics committees of the RI-MUHC (project identifier [ID] 2015-7656).

\section{Culture conditions}

Mycobacteria strains were grown in rolling liquid culture at $37^{\circ} \mathrm{C}$ in Middlebrook 7H9 (Difco) supplemented with $10 \%$ albumin dextrose catalase (ADC), $0.2 \%$ glycerol, and $0.05 \%$ Tween 80 (7H9 complete) or on $7 \mathrm{H} 10$ agar plates supplemented with $10 \%$ oleic acid ADC and $0.5 \%$ glycerol at $37^{\circ} \mathrm{C}$. When mentioned, the carbon source was modified from glycerol to acetate or the detergent Tween 80 was removed from the media. Bacillus cereus (clinical isolate), Corynebacterium glutamicum (ATCC 13032), Escherichia coli, and Pseudomonas aeruginosa PAO1 were grown in LB broth. HepG2 cells (ATCC HB-8065) were grown in Dulbecco's modified Eagle's medium (DMEM) with phenol red (from Gibco) supplemented with $10 \%$ fetal bovine serum at $37^{\circ} \mathrm{C}$ with $5 \% \mathrm{CO}_{2}$.

\section{Compound libraries}

The GSK library contains 176 small molecules with antimycobacterial activity, specifically against Mycobacterium tuberculosis [56]. Medicines for Malaria Venture created two compound sets: the Pathogen Box and the Pandemic Response Box. The Pathogen Box is composed of 400 molecules active against various disease sets (https://www.mmv.org/mmv-open/ pathogen-box/about-pathogen-box). The Pandemic Response Box is composed of 400 diverse drug-like molecules broadly categorized as antibacterials (201 molecules), antivirals (153 molecules), and antifungals (46 molecules) (https://www.mmv.org/mmv-open/pandemicresponse-box/about-pandemic-response-box).

\section{Library screening}

MMV Pathogen Box, MMV Pandemic Response Box, and GSK TB-active libraries were screened against $M$. abscessus ATCC 19977 strain which constitutively expressed the lux$C D A B E$ operon (M. abscessus lux) at $10 \mu \mathrm{M}$ in duplicate in 96-well flat-bottom plates in 7H9 complete media. The culture was grown to $\log$ phase $\left(\mathrm{OD}_{600} 0.4-0.8\right)$ and diluted to $\mathrm{OD}_{600}$ $0.005\left(5 \times 10^{6} \mathrm{CFU} / \mathrm{mL}\right) .90 \mu \mathrm{L}$ of culture was mixed with $10 \mu \mathrm{L}$ of compound. Plates were sealed with parafilm and incubated at $37^{\circ} \mathrm{C}$ for 48 hours. Plates had a column of $0.1 \% \mathrm{DMSO}$ as negative controls (drug-free conditions) and $64 \mu \mathrm{g} / \mathrm{mL}$ of AMK as positive controls. Luminescence was measured with an Infinite F200 Tecan plate reader. \% Luminescence relative to DMSO control was plotted using GraphPad Prism version 9. Compounds that decreased luminescence $\leq 10 \%$ were classified as primary hits. Primary hits were screened against $M$. 
abscessus ATCC 19977 at $10 \mu \mathrm{M}$ in triplicate using REMA (see "Determination of MIC" below). Plates were setup as performed for the primary screen. Fluorescence was measured with an Infinite F200 Tecan plate reader. \% bacteria viability relative to DMSO control was plotted using GraphPad Prism version 9. Compounds that decreased bacteria viability $\leq 10 \%$ were classified as confirmed hits. Confirmed hits were followed up with dose-response curves to determine the minimum inhibitory concentration (MIC).

\section{Determination of minimum inhibitory concentrations (MIC)}

MIC values were determined using the resazurin microtiter assay (REMA). Cultures were grown to log phase $\left(\mathrm{OD}_{600}\right.$ of $\left.0.4-0.8\right)$ and diluted to $\mathrm{OD}_{600}$ of 0.005 . Drugs were prepared in two-fold serial dilutions in 96-well plates with $90 \mu \mathrm{L}$ of bacteria per well to a final volume of $100 \mu \mathrm{L}$. Plates were incubated at $37^{\circ} \mathrm{C}$ until drug-free wells were turbid ( 2 days for M. abscessus). Ten $\mu \mathrm{L}$ of resazurin $(0.025 \% \mathrm{wt} / \mathrm{vol})$ was added to each well. Once the drug-free wells turned pink (one doubling time), the fluorescence (ex/em $560 \mathrm{~nm} / 590 \mathrm{~nm}$ ) was measured using an Infinite F200 Tecan plate reader. Fluorescence intensities were converted to \% viable cells relative to drug-free conditions and fit to the Gompertz equation using GraphPad Prism version 9. MIC values at $90 \%$ growth inhibition were determined from the nonlinear regression Gompertz equation.

\section{In vitro cytotoxicity in HepG2 cells}

Drugs were prepared in two-fold serial dilutions in 96-well plates with $45 \mu \mathrm{L}$ of Human HepG 2 cells $(2,000$ cells/well) to a final volume of $50 \mu \mathrm{L}$. Plates were incubated for 3 days at $37^{\circ} \mathrm{C}$ with $5 \% \mathrm{CO}_{2}$. Five $\mu \mathrm{L}$ of resazurin $(0.025 \% \mathrm{wt} / \mathrm{vol})$ was added to each well and incubated for 4 hours at $37^{\circ} \mathrm{C}$. Cell viability was determined from the fluorescence intensity as done in the previous REMA assay. $50 \%$ toxic dose concentrations $\left(\mathrm{TD}_{50}\right)$ values were obtained using a nonlinear regression fit equation ([inhibitor] vs response, variable slope) in GraphPad Prism version 9.

\section{Assessment of EPT efficacy in infected Zebrafish}

Experiments in zebrafish were conducted according to the Comité d'Ethique pour l'Expérimentation Animale de la Région Languedoc Roussillon under reference 2020022815234677V3. Experiments were performed using the golden mutant and macrophage reporter $\operatorname{Tg}(m p e g 1: m$ Cherry) lines as previously described [57]. Embryos were obtained and maintained as described [27]. Embryo age is expressed as hours post fertilisation (hpf). Green fluorescent $M$. abscessus CIP $104536^{\mathrm{T}}$ (R) expressing Wasabi were prepared and microinjected in the caudal vein $(2-3 \mathrm{~nL}$ containing $\approx 100$ bacteria/nL) in $30 \mathrm{hpf}$ embryos previously dechorionated and anesthetized with tricaine [58]. The bacterial inoculum was checked a posteriori by injection of $2 \mathrm{~nL}$ in sterile $\mathrm{PBS}^{\mathrm{T}}$ and plating on $7 \mathrm{H} 10^{\mathrm{OADC}}$. Infected embryos were transferred into 6-well plates (12 embryos/well) and incubated at $28.5^{\circ} \mathrm{C}$ to monitor kinetics of infection and embryo survival. Survival curves were determined by counting dead larvae daily for up to 11 days, with the experiment concluded when uninfected embryos started to die. EPT treatment of infected embryos and uninfected embryos was commenced at 24 hpi (hours post-infection) for 5 days. The drug-containing solution was renewed daily. Bacterial loads in live embryos were determined by anesthetising embryos in tricaine as previously described [59], mounting on 3\% (w/v) methylcellulose solution and taking fluorescent images using a Zeiss Axio Zoom.V16 coupled with an Axiocam 503 mono (Zeiss). Fluorescence Pixel Count (FPC) measurements were determined using the 'Analyse particles' function in Image [58]. All experiments were completed at least three times independently. 


\section{Isolation of resistant mutants}

Early $\log$ phase $M$. abscessus $\left(\mathrm{OD}_{600} 0.1-0.4\right)$ was cultured and resuspended to an $\mathrm{OD}_{600}$ of 10 . Ten $\mathrm{mL}$ of culture was kept as a reference strain for future sequencing. One hundred $\mu \mathrm{L}$ of $\mathrm{OD}_{600} 10$ (approx. $1 \mathrm{X} 10^{9} \mathrm{CFU} / 100 \mu \mathrm{L}$ ) was plated on solid media containing 10X, 20X, or $40 \mathrm{X} \mathrm{MIC}{ }_{90}$ of the compound of interest. AMK was used as a control antibiotic. Plates were incubated for 5 days at $37^{\circ} \mathrm{C}$. Number of colonies were counted to obtain mutation frequencies. Resistant colonies were transferred into fresh media without antibiotics (to avoid the emergence of secondary mutations). The MIC (REMA method) was measured for the mutant against the compound of interest as well as a panel of reference compounds with different targets for negative controls.

\section{Sequencing resistant mutants}

Genomic DNA (gDNA) was extracted from the $10 \mathrm{~mL}$ reference aliquot of M. abscessus harvested during mutant isolation and fresh cultures of mutant strains with confirmed resistance (REMA method) using the Qiagen QIAamp UCP Pathogen Mini kit with a modified mechanical lysis protocol. Pellet culture by centrifugation and resuspend in $590 \mu \mathrm{L}$ of ATL buffer containing the Dx reagent in a low-bind tube. Add $40 \mu \mathrm{L}$ of proteinase $\mathrm{K}(20 \mathrm{mg} / \mathrm{mL})$ and $20 \mu \mathrm{L}$ of lysozyme $(100 \mathrm{mg} / \mathrm{mL})$ and incubate for 30 minutes at $56^{\circ} \mathrm{C}$ under agitation. Transfer into a Pathogen Lysis Tube L and vortex twice using a FastPrep-24 instrument at $6.5 \mathrm{~m} / \mathrm{s}$ for $45 \mathrm{~s}$ with a 5-minute incubation on ice in between. Transfer supernatant into fresh $2 \mathrm{~mL}$ low-bind tube. Follow manufacturer's instructions for sample prep with spin protocol. gDNA was quantified with Quant-iT PicoGreen dsDNA kit. leuS from ATCC 19977 and EPT mutants was sequenced with Sanger Sequencing with the primers listed in S5 Table.

\section{Cloning and over-expressing mutant leuS in M. abscessus}

Wildtype and mutant leuS (D436H) were PCR amplified from gDNA using Phusion with primers 1 \& 2 (see S5 Table), ligated into pMV306_hsp60 digested with EcoRV and HindIII restriction enzymes, and transformed into E. coli DH5 $\alpha$ cells (Promega). Plasmids were extracted and sequenced with primers 3-12 (see S5 Table) using Sanger sequencing.

\section{Protein purification}

The M. abscessus LeuRS editing domain (residues 303-499 of WP005112800.1) and the $M$. tuberculosis LeuRS editing domain (residues 311-512 of WP_003900794.1) were synthesized with an N-terminal polyhistidine tag and tobacco etch virus (TEV) protease recognition site, with codon optimization for E. coli and cloned into pET24a(+) by the company BioBasic to create pMabsED and pMtbED, respectively. E. coli BL21 (DE3) cells were transformed with pMabsED or pMtbED and grown overnight at $37^{\circ} \mathrm{C}$ on LB-agar with $50 \mu \mathrm{g} / \mathrm{mL}$ kanamycin. Single colonies were transferred into $100 \mathrm{~mL}$ of LB media with $50 \mu \mathrm{g} / \mathrm{mL}$ kanamycin (LB-kan) and grown overnight at $37^{\circ} \mathrm{C}$. One liter of LB-kan was inoculated with $10 \mathrm{~mL}$ of the overnight culture and grown at $37^{\circ} \mathrm{C}$ until an optical density at $600 \mathrm{~nm}$ of 0.6 was reached. Protein expression was then induced with addition of $0.5 \mathrm{mM}$ IPTG and the culture further incubated for $18 \mathrm{~h}$ at $16^{\circ} \mathrm{C}$. The cells were harvested by centrifugation ( $4000 \mathrm{~g}$ for $20 \mathrm{~min}$ ), resuspended in buffer A ( $50 \mathrm{mM}$ Tris, $150 \mathrm{mM} \mathrm{NaCl}, 2 \mathrm{mM} \mathrm{BME}$ ) plus $2 \mathrm{mM} \mathrm{PMSF}$, lysed using sonication (55\% amplitude, 30 cycles of $10 \mathrm{~s}$ on $20 \mathrm{~s}$ off) and the lysate clarified by ultracentrifugation $(40,000 \mathrm{~g}$ for $30 \mathrm{~min})$. The lysate was loaded onto a HiTrap FF (Cytiva) and eluted with buffer A plus $500 \mathrm{mM}$ imidazole. The eluate was dialyzed in buffer A, digested with TEV protease overnight and the protease as well as non-cleaved protein separated from cleaved protein by 
application to the HiTrap FF column with flow-through collected. Protein was then subjected to size exclusion chromatography using a HiLoad Superdex Increase 75 colum (Cytiva), with fractions containing pure protein pooled.

\section{Isothermal titration calorimetry}

ITC measurements were performed at $30^{\circ} \mathrm{C}$ on a VP-ITC system (Malvern Panalytical Inc). Epetraborole $(100 \mu \mathrm{M})$ in measurement buffer ( $50 \mathrm{mM}$ Tris, $150 \mathrm{mM} \mathrm{NaCl}, 2 \mathrm{mM} \mathrm{BME}, 10$ mM AMP) was titrated with protein solution $(1 \mathrm{mM})$ in measurement buffer over 29 injections of $10 \mu \mathrm{l}$ with 300 seconds equilibration time between injections. The heat evolved after each protein injection was calculated by integrating the calorimetric signal. The binding isotherms obtained were fitted to a single-site model using Origin 7 (Microcal Inc.). Experiments were performed in triplicates.

\section{Crystallography}

Initial crystals of the M. abscessus LeuRS editing domain were obtained from sparse matrix screening in 96-well sitting drop format using a sample of $10 \mathrm{mg} / \mathrm{mL}$ protein in buffer $\mathrm{A}$, and a precipitant solution of $100 \mathrm{mM}$ HEPES $\mathrm{pH} 7.0$ and $2 \mathrm{M}$ ammonium sulfate, at room temperature. Diffraction-quality crystals were grown by mixing $2 \mu \mathrm{L}$ of protein solution $(7.5 \mathrm{mg} / \mathrm{mL})$ and $2 \mu \mathrm{L}$ of reservoir solution (100 mM HEPES pH 7.0 and 2.5 M ammonium sulfate) in 24-well sittingdrop format. Crystals were cryoprotected in $100 \mathrm{mM}$ HEPES pH 7.0 and 3.5 $\mathrm{M}$ ammonium sulfate, looped and flash-vitrified in liquid nitrogen. Diffracting co-crystals of the editing domain and epetraborole were obtained by streak seeding un-liganded crystals shards into drops in 24-well sitting-drop format with a reservoir solution of $100 \mathrm{mM}$ HEPES, pH 7.5, 2\% PEG400, $2.1 \mathrm{M}$ ammonium sulfate, $10 \mathrm{mM}$ AMP, $1 \mathrm{mM}$ epetraborole and $15 \%$ glycerol at room temperature. These crystals were directly looped and flash-vitrified in liquid nitrogen for diffraction experiments.

\section{Structure determination}

Diffraction data for the unliganded editing domain was collected at the Advanced Photon Source (24-ID-E) and data for the epetraborole-editing domain complex at the Canadian Light Source (CMCF-08B1-1). The data sets were indexed, processed, and scaled with HKL2000 [60] (for the unliganded editing domain) or DIALS [61] (for the complex). Initial phases for the $M$. abscessus LeuRS editing domain were obtained using molecular replacement with the program Phaser [62] using 5AGR [19] as a search model. Iterative rounds of refinement with Phenix [63] and manual modeling in Coot [64] yielded the final unliganded structure. The structure of the M. abscessus LeuRS editing domain with epetraborole bound in the active site crystallized in a different crystal form (S5 Table). Therefore, initial phases for the co-complex structure were obtained with molecular replacement using the program Phaser [62] utilizing the unliganded structure as a search model. The model for epetraborole was generated with eLBOW [65] and geometrical restraints were obtained with AceDRG [66]. Iterative rounds of refinement using Phenix [63] and manual modeling in Coot [64] yielded the final co-complex structure.

\section{Time-kill assays}

Log phase $\left(\mathrm{OD}_{600}\right.$ of $\left.0.4-0.8\right)$ M. abscessus was diluted to an $\mathrm{OD}_{600}$ of $0.0001\left(10^{5} \mathrm{CFU} / \mathrm{mL}\right)$ and incubated with drugs of interest. One hundred $\mu \mathrm{L}$ aliquots were taken and serially diluted in 7H9 complete and plated on 7H10 agar. The starting inoculum was determined from time 0 before drugs were added. CFUs were determined after 4 days of incubation at $37^{\circ} \mathrm{C}$. Bactericidal activity is defined as a $3 \log _{10}$ decrease $(99.9 \%)$ from the starting inoculum. 


\section{Checkerboard assays}

Drug combinations were assessed for synergy, indifference, or antagonism using the standard checkerboard format followed by REMA for MIC determination. Fractional inhibitory concentrations (FICs): FIC $(\mathrm{X}+\mathrm{Y})=(\mathrm{MIC}$ of $\mathrm{X}$ in combination with $\mathrm{Y}) /(\mathrm{MIC}$ of $\mathrm{X}$ alone). The fractional inhibitory concentration index (FICI) was calculated from FIC $_{X}+$ FIC $_{Y}$. FICI values $<0.5$ are defined as synergy, FICI values $\geq 4.0$ are defined as antagonism, and FICI values in between are defined as indifferent. EPT was serially diluted two-fold from 4X MIC to 1/ 16 X MIC. Mycobacterial drugs were serially diluted two-fold from 8X MIC to 1/32X MIC.

\section{Inducible resistance assay}

To test whether M. abscessus has inducible resistance to EPT, we performed a preexposure assay. Briefly, log phase $M$. abscessus was diluted to 0.05 and grown overnight with $1 / 4 \mathrm{X} \mathrm{MIC}_{50}$ of EPT $(0.016 \mu \mathrm{g} / \mathrm{mL})$ and CLR $(0.016 \mu \mathrm{g} / \mathrm{mL})$. After overnight growth, MICs were determined via REMA. Data is reported as the ratio of pre-exposed culture MIC to MIC of culture grown in drug-free conditions.

\section{CRISPRi conditional knockdown of leuS}

The PLJR962 plasmid was restriction digested with BsmBI and gel purified. Synthetic oligo primers (S4 Table) for the sgRNA with the PAM sequence for leuS were annealed and ligated into digested PLJR962 vector and transformed into E. coli DH5 $\alpha$. Clones were sequenced using sequencing primer (S4 Table). $500 \mathrm{ng}$ of CRISPRi leuS vector was electroporated into M. abscessus ATCC 19977. Colonies were picked from $7 \mathrm{H} 10$ plates containing $250 \mu \mathrm{g} / \mathrm{mL}$ kanamycin and confirmed by PCR. M. abscessus CRISPRi leuS was grown in liquid culture to early log phase $\left(\mathrm{OD}_{600} 0.1-0.4\right)$ and diluted to $1 \times 10^{4} \mathrm{CFU} / \mathrm{mL}$. One hundred $\mu \mathrm{L}$ of culture was plated on $7 \mathrm{H} 10$ containing either serial dilutions of EPT or RFB as control, and $0,0.05 \mu \mathrm{g} / \mathrm{mL}$, or $0.1 \mu \mathrm{g} /$ $\mathrm{mL}$ ATc, the inducer of the sgRNA and catalytically inactive Cas9 (dCas9). The $\mathrm{MIC}_{99}$ is the concentration of EPT or RFB that prevented growth relative to the drug free plate.

\section{Norvaline suppression of mutants}

Early $\log$ phase $M$. abscessus or $M$. tuberculosis $\left(\mathrm{OD}_{600} 0.1-0.4\right)$ was cultured and resuspended to an $\mathrm{OD}_{600}$ of 10 (M. abscessus) or $\mathrm{OD}_{600}$ of 1 (M. tuberculosis). One hundred $\mu \mathrm{L}$ of culture (approx. 1 X $10^{9} \mathrm{CFU}$ for M. abscessus and $1 \times 10^{6} \mathrm{CFU}$ for M. tuberculosis) was plated on solid media containing $10 \mathrm{X} \mathrm{MIC}_{90} \mathrm{EPT}, 10 \mathrm{X} \mathrm{MIC} \mathrm{C}_{90} \mathrm{EPT}+590 \mu \mathrm{g} / \mathrm{mL}$ norvaline, or $590 \mu \mathrm{g} / \mathrm{mL}$ norvaline. The experiment was repeated with $10 \mathrm{X} \mathrm{MIC}_{90} \mathrm{RFB}$ as control. M. abscessus plates were incubated for 5 days at $37^{\circ} \mathrm{C}, M$. tuberculosis plates were incubated for 5 weeks at $37^{\circ} \mathrm{C}$. Number of colonies were enumerated to obtain mutation frequencies. Since, mutations are considered rare events, mutation frequency rates between $10 \mathrm{X} \mathrm{MIC} \mathrm{C}_{90} \mathrm{EPT}$ and $10 \mathrm{X} \mathrm{MIC}_{90} \mathrm{EPT}$ $+590 \mu \mathrm{g} / \mathrm{mL}$ norvaline were compared using the two Poisson rates.

The Poisson rate is defined as the number of events divided by the sample size: $\lambda=X / N$ The rates were compared using the small sample z-test:

$$
z_{S R}=\frac{\sqrt{\lambda_{2}}-\sqrt{\lambda_{1}}}{\frac{1}{2} \sqrt{\frac{1}{N_{1}}+\frac{1}{N_{2}}}}
$$

\section{LC-MS/MS measurement of norvaline in the proteome}

Wildtype, mutant, and complement $M$. abscessus strains were grown for $12 \mathrm{~h}$ or 3 days in $59 \mu \mathrm{g} / \mathrm{mL}$ norvaline or $59 \mu \mathrm{g} / \mathrm{mL}$ valine. Proteins were extracted using an optimized protocol 
for mass spectrometry follow-up [67]. Cells were collected, washed with ice-cold PBS, and resuspended in $1 \mathrm{~mL}$ lysis buffer $\left(50 \mathrm{mM} \mathrm{NH}_{4} \mathrm{HCO}_{3} \mathrm{pH} 7.4,10 \mathrm{mM} \mathrm{MgCl}_{2}, 0.1 \% \mathrm{NaN}_{3}, 1 \mathrm{mM}\right.$ EGTA, 1 x protease inhibitors (Roche), $7 \mathrm{M}$ urea, and $2 \mathrm{M}$ thiourea). Cells were lysed with zirconia beads and the cell lysate was collected and filtered through a $0.22 \mu \mathrm{m}$ membrane. Proteins were precipitated overnight at $4{ }^{\circ} \mathrm{C}$ with TCA at $25 \%(\mathrm{v} / \mathrm{v})$. The precipitate was washed with $1 \mathrm{~mL}$ cold acetone and $250 \mu \mathrm{L}$ cold water. The final wash is only water. The pellet was resuspended in $200 \mu \mathrm{L}$ resuspension buffer $\left(50 \mathrm{mM} \mathrm{NH}_{4} \mathrm{HCO}_{3}, 1 \mathrm{M}\right.$ urea). Protein extraction was quantified with the Bradford assay and the quality of proteins was examined on SDS-PAGE. Protein lysates were dissolved in SDS-PAGE reducing buffer and electrophoresed onto a single stacking gel band to remove lipids, detergents and salts. For each sample, a single gel band was reduced with DTT (Sigma), alkylated with iodoacetic acid (Sigma) and digested with LCMS grade trypsin (Promega). Extracted peptides were re-solubilized in $0.1 \%$ aqueous formic acid and loaded onto a Thermo Acclaim Pepmap (Thermo, $75 \mu \mathrm{M}$ ID X $2 \mathrm{~cm} \mathrm{C18}$ $3 \mu \mathrm{M}$ beads) precolumn and then onto an Acclaim Pepmap Easyspray (Thermo, $75 \mu \mathrm{M} \mathrm{X} 15$ $\mathrm{cm}$ with $2 \mu \mathrm{M}$ C18 beads) analytical column separation using a Dionex Ultimate $3000 \mathrm{uHPLC}$ at $250 \mathrm{nl} / \mathrm{min}$ with a gradient of $2-35 \%$ organic ( $0.1 \%$ formic acid in acetonitrile) over 2 hours. Peptides were analyzed using a Thermo Orbitrap Fusion mass spectrometer operating at 120,000 resolution for MS1 with HCD sequencing at top speed (15,000 resolution) for all peptides with a charge of $2+$ or greater. The raw data were converted into ${ }^{*}$.mgf format (Mascot generic format) for searching using the Mascot 2.5.1 search engine (Matrix Science) against Mycobacterium abscessus protein sequences (Uniprot downloaded 2020.11.30). A modification for Xle->Val was used to detect incorporation of Val into WT sequences. The database search results were loaded onto Scaffold Q+ Scaffold_4.4.8 (Proteome Sciences) for statistical treatment and data visualization.

\section{Murine model of chronic M. abscessus infection using NOD.CB17- Prkdc $^{\text {scid }} / \mathrm{NCrCrl}$ mice}

RFB (Sigma), EPT (Cayman Chemical), and norvaline (Sigma) were dissolved in $0.5 \% \mathrm{w} / \mathrm{v}$ sterile low viscosity carboxymethyl cellulose $\mathrm{pH} 7$ (vehicle). Drugs were aliquoted and stored at $-20^{\circ} \mathrm{C}$ for the duration of the 10-day treatment. 6-8 weeks-old female mice were ordered from Charles River Labs. Mice were intranasally infected with $\sim 10^{6} \mathrm{CFU}\left(25 \mu \mathrm{L}\right.$ of $5 \times 10^{8} \mathrm{CFU} /$ $\mathrm{mL}$ ) of M. abscessus CIP $104536^{\mathrm{T}}$ (rough morphotype). Five mice were sacrificed 4 hours postinfection to determine initial lung inoculum and on day 1 to enumerate CFU prior to drug administration. Mice were treated daily by oral gavage with $100 \mu \mathrm{L}$ of vehicle or drug. On day 11 , mice were humanely euthanized, the lungs were collected and homogenized in $1 \mathrm{~mL}$ of $7 \mathrm{H} 9$ complete. Lung homogenates were plated on 7H11 agar and plates were incubated at $37^{\circ} \mathrm{C}$ for 5 days. CFU data was log-transformed for one-way ANOVA with Tukey's multiple comparisons test using GraphPad Prism version 9.

\section{Supporting information}

S1 Fig. M. abscessus phenotypic screening with $176 \mathrm{~TB}$-active compounds from GSK

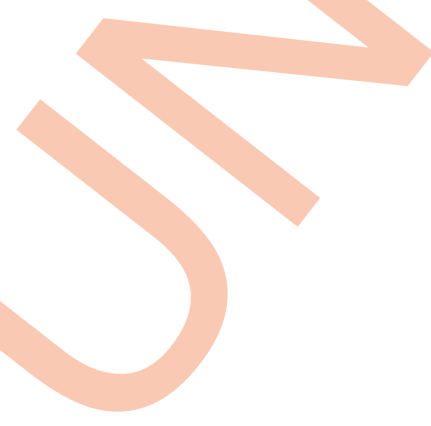
(A-C) and MMV (D-F). A Luminescence relative to drug free conditions using M. abscessus lux. Data is shown in duplicate. B-C Secondary screening of primary hits using REMA on $M$. abscessus ATCC 19977. \% bacteria viability relative to drug free conditions. Data is shown as mean \pm SD from technical triplicates. Dashed red line indicates $10 \%$ viability threshold when screened at $10 \mu \mathrm{M}$. Three hits were identified from primary screen however, all three did not pass the secondary screen. No active compounds were identified from this library. D MMV Pathogen box and $\mathbf{E}$ Pandemic response box; luminescence relative to drug free conditions 
using M. abscessus lux. Data is shown in duplicate. F Secondary screening of primary hits using REMA on M. abscessus ATCC 19977. \% bacteria viability relative to drug free conditions. Data is shown in technical triplicate. Dashed red line indicates $10 \%$ viability threshold when screened at $10 \mu \mathrm{M}$. G Correlation between hits from luminescence primary screen and bacterial viability secondary screen. Correlation calculated using non-parametric Spearman correlation. 20 compounds passed the primary screen and 9 passed the secondary screen. Only three compounds were still active after acquiring fresh batch and displayed dose-dependent activity (3/ 800). EPT in cyan. AMK in blue as positive control. Two negative hits from luminescence screen in magenta used as negative controls. Drug free in black used as media control. (TIFF)

S2 Fig. Isobolograms for potential combination therapy with EPT. Green area indicates synergy (FICI $<0.5$ ); red area indicates antagonism (FICI $\geq 4.0$ ); white area indicates indifferent. RIF and CLR, and AMK and CLR are used as synergy and antagonism controls, respectively. Data shown is from checkerboard assays done in technical duplicate. (TIFF)

S3 Fig. Drug susceptibility assessment of resistant mutants. A-D Dose-response curves of isolated EPT (10X, 20X, 40X MIC) mutants or AMK (40X MIC) mutant. AMK was used as control compound for mutant isolation. RIF and BDQ were used for cross-resistance verification. ATCC 19977 (black circles), D436H mutant-1 (yellow squares), D436H mutant-2 (pink diamonds), D436H mutant-3 (green triangles), D436H mutant-4 (purple inverted triangles), AMK 40X (crosses). Data is mean \pm SD from two independent experiments.

S4 Fig. Thermodynamic analysis of EPT binding to LeuRS editing domain. Heat of injection (upper panel) and single-site binding model of the integrated isotherm (lower panel). Mabs LeuRS (left) or Mtb LeuRS (right) editing domains bound to EPT.

(TIFF)

S5 Fig. Co-crystal structure of LeuRS and benzoxaborole adducts. A Difference maps for

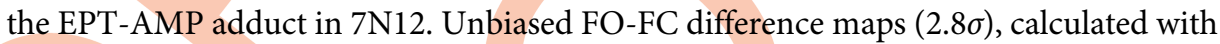
phases from a model that never included ligand. B Comparison of apo (green, PDB 7N11) and co-complex (cyan, PDB 7N12) structures of M. abscessus LeuRS bound to EPT-AMP. C Benzoxaborole inhibitors of LeuRS. (Left) EPT-AMP adduct bound to M. abscessus LeuRS. (Right) BNZ-AMP adduct bound to M. tuberculosis LeuRS.

(TIFF)

S1 Table. Carbon dependent variability on EPT activity. G, Glycerol; A, Acetate; Tw80, Tween-80; CaMH, Cation-adjusted Muller-Hinton. (DOCX)

S2 Table. Epetraborole in vitro activity against clinical isolates. ${ }^{\mathrm{a}}$ Morphology as determined by smooth (S) or rough (R) colonies on 7H10 agar. Drugs used: EPT, Epetraborole; AMK, Amikacin; RIF, Rifampicin; BDQ, Bedaquiline; CFX, Cefoxitin; CLR, Clarithromycin. (DOCX)

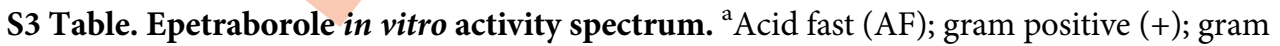
negative (-). EPT, Epetraborole; AMK, Amikacin; RIF, Rifampicin; ERV, BDQ, Bedaquiline; CFX, Cefoxitin; CLR, Clarithromycin. Species: Mycobacterium abscessus, Mycobacterium avium hominissuis, Mycobacterium avium intracellulaire, Mycobacterium tuberculosis H37Rv, Mycobacterium tuberculosis Erdman, Bacillus cereus, Corynebacterium glutamicum, 
Escherichia coli, Pseudomonas aeruginosa.

(DOCX)

S4 Table. In vitro resistance frequency. ${ }^{\mathrm{a}} \mathrm{N} . \mathrm{D}$ : not determined. ${ }^{\mathrm{b}} \mathrm{MIC} \mathrm{C}_{90}$ values on $7 \mathrm{H} 10$ agar used in this experiment were $4.0 \mu \mathrm{g} / \mathrm{mL}$ and $0.27 \mu \mathrm{g} / \mathrm{mL}$ for amikacin and epetraborole, respectively.

(DOCX)

S5 Table. Primers.

(DOCX)

S6 Table. Whole genome sequencing variants identified in EPT-resistant M. abscessus. (DOCX)

S7 Table. Data collection and refinement statistics. (DOCX)

\section{Acknowledgments}

We thank Adam Hassan (Research Institute of the McGill University Health Centre) for technical assistance with mouse experiments. We thank Medicines for Malaria Venture for providing the MMV Open pathogen box and pandemic response boxes. This research used resources of the Advanced Photon Source, a U.S. Department of Energy (DOE) Office of Science User Facility, operated for the DOE Office of Science by Argonne National Laboratory under Contract No. $D E-A C 02-06 C H 11357$. Extraordinary facility operations were supported in part by the DOE Office of Science through the National Virtual Biotechnology Laboratory, a consortium of DOE national laboratories focused on the response to COVID-19, with funding provided by the Coronavirus CARES Act. Part or all of the research described in this paper was performed using beamline CMCF-BM at the Canadian Light Source, a national research facility of the University of Saskatchewan, which is supported by the Canada Foundation for Innovation (CFI), the Natural Sciences and Engineering Research Council (NSERC), the National Research Council (NRC), the Canadian Institutes of Health Research (CIHR), the Government of Saskatchewan, and the University of Saskatchewan. The luxCDABE plasmid was kindly gifted by Jeffery S.

Cox. We are grateful to the CRBM zebrafish facility (Montpellier), P. Richard and M. Plays for zebrafish husbandry.

\section{Author Contributions}

Conceptualization: Jaryd R. Sullivan, Marcel A. Behr.

Formal analysis: Jaryd R. Sullivan, Elias Kalthoff, Claire Hamela, Lorne Taylor.

Funding acquisition: Marcel A. Behr.

Investigation: Jaryd R. Sullivan, Andréanne Lupien, Elias Kalthoff, Claire Hamela, Lorne Taylor, Kim A. Munro.

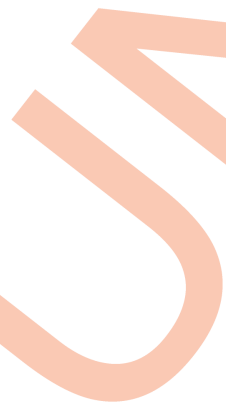

Methodology: Jaryd R. Sullivan, Elias Kalthoff, Claire Hamela.

Project administration: Marcel A. Behr.

Resources: T. Martin Schmeing, Laurent Kremer, Marcel A. Behr.

Supervision: Andréanne Lupien, T. Martin Schmeing, Laurent Kremer, Marcel A. Behr.

Visualization: Jaryd R. Sullivan, Claire Hamela, T. Martin Schmeing. 
Writing - original draft: Jaryd R. Sullivan, Marcel A. Behr.

Writing - review \& editing: Jaryd R. Sullivan, Andréanne Lupien, T. Martin Schmeing, Laurent Kremer, Marcel A. Behr.

\section{References}

1. Johansen MD, Herrmann J-L, Kremer L. Non-tuberculous mycobacteria and the rise of Mycobacterium abscessus. Nat Rev Microbiol. 2020. https://doi.org/10.1038/s41579-020-0331-1 PMID: 32086501

2. Griffith DE, Aksamit T, Brown-Elliott BA, Catanzaro A, Daley C, Gordin F, et al. An official ATS/IDSA statement: Diagnosis, treatment, and prevention of nontuberculous mycobacterial diseases. Am J Respir Crit Care Med. 2007; 175: 367-416. https://doi.org/10.1164/rccm.200604-571ST PMID: 17277290

3. Haworth CS, Banks J, Capstick T, Fisher AJ, Gorsuch T, Laurenson IF, et al. British Thoracic Society guidelines for the management of non-tuberculous mycobacterial pulmonary disease (NTM-PD). Thorax. 2017; 72: ii1-ii64. https://doi.org/10.1136/thoraxjnl-2017-210927 PMID: 29054853

4. Lee MR, Sheng WH, Hung CC, Yu CJ, Lee LN, Hsueh PR. Mycobacterium abscessus complex infections in humans. Emerg Infect Dis. 2015; 21: 1638-1646. https://doi.org/10.3201/2109.141634 PMID: 26295364

5. Van Ingen J, Boeree MJ, Van Soolingen D, Mouton JW. Resistance mechanisms and drug susceptibility testing of nontuberculous mycobacteria. Drug Resist Updat. 2012; 15: 149-161. https://doi.org/10. 1016/j.drup.2012.04.001 PMID: 22525524

6. Wu ML, Aziz DB, Dartois V, Dick T. NTM drug discovery: status, gaps and the way forward. Drug Discov Today. 2018; 0: 1-18. https://doi.org/10.1016/j.drudis.2018.04.001 PMID: 29635026

7. Kim TS, Choe JH, Kim YJ, Yang CS, Kwon HJ, Jeong J, et al. Activity of LCB01-0371, a novel oxazolidinone, against mycobacterium abscessus. Antimicrob Agents Chemother. 2017;61. https://doi.org/10. 1128/AAC.02752-16 PMID: 28674049

8. Le Run E, Arthur M, Mainardia JL. In Vitro and Intracellular Activity of Imipenem Combined with Tedizolid, Rifabutin, and Avibactam against Mycobacterium abscessus. Antimicrob Agents Chemother. 2019;63. https://doi.org/10.1128/AAC.01915-18 PMID: 30745387

9. Dupont C, Viljoen A, Dubar F, Blaise M, Bernut A, Pawlik A, et al. A new piperidinol derivative targeting mycolic acid transport in Mycobacterium abscessus. Mol Microbiol. 2016; 101: 515-529. https://doi.org/ 10.1111/mmi.13406 PMID: 27121350

10. Kozikowski AP, Onajole OK, Stec J, Dupont C, Viljoen A, Richard M, et al. Targeting Mycolic Acid Transport by Indole-2-carboxamides for the Treatment of Mycobacterium abscessus Infections. J Med Chem. 2017; 60: 5876-5888. https://doi.org/10.1021/acs.jmedchem.7b00582 PMID: 28574259

11. Rock FL, Mao W, Yaremchuk A, Tukalo M, Crépin T, Zhou H, et al. An antifungal agent inhibits an aminoacyl-tRNA synthetase by trapping tRNA in the editing site. Science (80-). 2007; 316: 1759-1761. https://doi.org/10.1126/science.1142189 PMID: 17588934

12. Baker SJ, Zhang YK, Akama T, Lau A, Zhou H, Hernandez V, et al. Discovery of a new boron-containing antifungal agent, 5-fluoro-1,3-dihydro-1-hydroxy-2,1-benzoxaborole (AN2690), for the potential treatment of onychomycosis. J Med Chem. 2006; 49: 4447-4450. https://doi.org/10.1021/jm0603724 PMID: 16854048

13. Nureki O, Vassylyev DG, Tateno M, Shimada A, Nakama T, Fukai S, et al. Enzyme structure with two catalytic sites for double-sieve selection of substrate. Science (80-). 1998; 280: 578-582. https://doi. org/10.1126/science.280.5363.578 PMID: 9554847

14. Nangle LA, Motta CM, Schimmel P. Global Effects of Mistranslation from an Editing Defect in Mammalian Cells. Chem Biol. 2006; 13: 1091-1100. https://doi.org/10.1016/j.chembiol.2006.08.011 PMID: 17052613

15. Penn BH, Netter Z, Johnson JR, Von Dollen J, Jang GM, Johnson T, et al. An Mtb-Human Protein-Protein Interaction Map Identifies a Switch between Host Antiviral and Antibacterial Responses. Mol Cell. 2018; 71: 637-648.e5. https://doi.org/10.1016/j.molcel.2018.07.010 PMID: 30118682

16. Pethe K, Sequeira PC, Agarwalla S, Rhee K, Kuhen K, Phong WY, et al. A chemical genetic screen in Mycobacterium tuberculosis identifies carbon-source-dependent growth inhibitors devoid of in vivo efficacy. Nat Commun. 2010; 1: 57. https://doi.org/10.1038/ncomms1060 PMID: 20975714

17. Aziz DB, Low JL, Wu ML, Gengenbacher M, Teo JWP, Dartois V, et al. Rifabutin Is active against mycobacterium abscessus complex. Antimicrob Agents Chemother. 2017;61. https://doi.org/10.1128/AAC 00155-17 PMID: 28396540 
18. Fass RJ, Barnishan J. Effect of divalent cation concentrations on the antibiotic susceptibilities of nonfermenters other than Pseudomonas aeruginosa. Antimicrob Agents Chemother. 1979; 16: 434-438. https://doi.org/10.1128/AAC.16.4.434 PMID: 117746

19. Palencia A, Li X, Bu W, Choi W, Ding CZ, Easom EE, et al. Discovery of novel oral protein synthesis inhibitors of mycobacterium tuberculosis that target leucyl-tRNA synthetase. Antimicrob Agents Chemother. 2016; 60: 6271-6280. https://doi.org/10.1128/AAC.01339-16 PMID: 27503647

20. Maurer FP, Bruderer VL, Ritter C, Castelberg C, Bloemberg G V, Böttger EC. Lack of antimicrobial bactericidal activity in Mycobacterium abscessus. Antimicrob Agents Chemother. 2014; 58: 3828-3836. https://doi.org/10.1128/AAC.02448-14 PMID: 24752273

21. Richard M, Gutiérrez AV, Kremer L. Dissecting erm(41)-mediated macrolide-inducible resistance in mycobacterium abscessus. Antimicrob Agents Chemother. 2020; 64. https://doi.org/10.1128/AAC. 01879-19 PMID: 31791943

22. Nash KA, Brown-Elliott AB, Wallace RJ. A Novel gene, erm(41), confers inducible macrolide resistance to clinical isolates of mycobacterium abscessus but is absent from mycobacterium chelonae. Antimicrob Agents Chemother. 2009; 53: 1367-1376. https://doi.org/10.1128/AAC.01275-08 PMID: 19171799

23. Pryjma M, Burian J, Kuchinski K, Thompson CJ. Antagonism between Front-Line Antibiotics Clarithromycin and Amikacin in the Treatment of Mycobacterium abscessus Infections is Mediated by the whiB7 gene. Antimicrob Agents Chemother. 2017;61. https://doi.org/10.1128/AAC.01353-17 PMID: 28874379

24. Aziz DB, Go ML, Dick T. Rifabutin Suppresses Inducible Clarithromycin Resistance in Mycobacterium abscessus by Blocking Induction of whiB7 and erm41. Antibiotics. 2020;9. https://doi.org/10.3390/ antibiotics10010009 PMID: 33374204

25. Bernut A, Herrmann J-L, Ordway D, Kremer L. The Diverse Cellular and Animal Models to Decipher the Physiopathological Traits of Mycobacterium abscessus Infection. Front Cell Infect Microbiol. 2017;7. https://doi.org/10.3389/fcimb.2017.00007 PMID: 28164039

26. Bernut A, Dupont C, Ogryzko N V, Neyret A, Herrmann JL, Floto RA, et al. CFTR Protects against Mycobacterium abscessus Infection by Fine-Tuning Host Oxidative Defenses. Cell Rep. 2019; 26: 1828-1840.e4. https://doi.org/10.1016/j.celrep.2019.01.071 PMID: 30759393

27. Bernut A, Herrmann JL, Kissa K, Dubremetz JF, Gaillard JL, Lutfalla G, et al. Mycobacterium abscessus cording prevents phagocytosis and promotes abscess formation. Proc Natl Acad Sci U S A. 2014;111. https://doi.org/10.1073/pnas.1313318110 PMID: 24324143

28. Hernandez V, Crépin T, Palencia A, Cusack S, Akama T, Baker SJ, et al. Discovery of a novel class of boron-based antibacterials with activity against gram-negative bacteria. Antimicrob Agents Chemother. 2013; 57: 1394-1403. https://doi.org/10.1128/AAC.02058-12 PMID: 23295920

29. Lincecum TL, Tukalo M, Yaremchuk A, Mursinna RS, Williams AM, Sproat BS, et al. Structural and mechanistic basis of pre- and posttransfer editing by leucyl-tRNA synthetase. Mol Cell. 2003; 11: 951963. https://doi.org/10.1016/s1097-2765(03)00098-4 PMID: 12718881

30. O'Dwyer K, Spivak AT, Ingraham K, Min S, Holmes DJ, Jakielaszek C, et al. Bacterial resistance to leucyl-tRNA synthetase inhibitor GSK2251052 develops during treatment of complicated urinary tract infections. Antimicrob Agents Chemother. 2015; 59: 289-298. https://doi.org/10.1128/AAC.03774-14 PMID: 25348524

31. Rock JM, Hopkins FF, Chavez A, Diallo M, Chase MR, Gerrick ER, et al. Programmable transcriptional repression in mycobacteria using an orthogonal CRISPR interference platform. Nat Microbiol. 2017; 2: 16274. https://doi.org/10.1038/nmicrobiol.2016.274 PMID: 28165460

32. Zhai Y, Martinis SA. Two conserved threonines collaborate in the Escherichia coli Leucyl-tRNA synthetase amino acid editing mechanism. Biochemistry. 2005; 44: 15437-15443. https://doi.org/10.1021/ bi0514461 PMID: 16300391

33. Alvarez-Carreño C, Becerra A, Lazcano A. Norvaline and Norleucine May Have Been More Abundant Protein Components during Early Stages of Cell Evolution. Orig Life Evol Biosph. 2013; 43: 363-375. https://doi.org/10.1007/s11084-013-9344-3 PMID: 24013929

34. Ji QQ, Fang ZP, Ye Q, Chi CW, Wang ED. Self-protective responses to norvaline-induced stress in a leucyl-tRNA synthetase editing-deficient yeast strain. Nucleic Acids Res. 2017; 45: 7367-7381. https:// doi.org/10.1093/nar/gkx487 PMID: 28575390

35. Cvetesic N, Palencia A, Halasz I, Cusack S, Gruic-Sovulj I. The physiological target for Leu RS translational quality control is norvaline. EMBO J. 2014; 33: 1639-1653. https://doi.org/10.15252/embj. 201488199 PMID: 24935946

36. Bacher JM, Schimmel P. An editing-defective aminoacyl-tRNA synthetase is mutagenic in aging bacteria via the SOS response. Proc Natl Acad Sci U S A. 2007; 104: 1907-1912. https://doi.org/10.1073/ pnas.0610835104 PMID: 17264207 
37. Song Y, Zhou H, Vo MN, Shi Y, Nawaz MH, Vargas-Rodriguez O, et al. Double mimicry evades tRNA synthetase editing by toxic vegetable-sourced non-proteinogenic amino acid. Nat Commun. 2017;8. https://doi.org/10.1038/s41467-017-00021-9 PMID: 28364116

38. Lu J, Bergert M, Walther A, Suter B. Double-sieving-defective aminoacyl-tRNA synthetase causes protein mistranslation and affects cellular physiology and development. Nat Commun. 2014;5. https://doi org/10.1038/ncomms6650 PMID: 25427601

39. Lee JW, Beebe K, Nangle LA, Jang J, Longo-Guess CM, Cook SA, et al. Editing-defective tRNA synthetase causes protein misfolding and neurodegeneration. Nature. 2006; 443: 50-55. https://doi.org/10. 1038/nature05096 PMID: 16906134

40. Schimmel P. Mistranslation and its control by tRNA synthetases. Philos Trans R Soc B Biol Sci. 2011; 366: 2965-2971. https://doi.org/10.1098/rstb.2011.0158 PMID: 21930589

41. Dick T, Shin SJ, Koh WJ, Dartois V, Gengenbacher M. Rifabutin is active against mycobacterium abscessus in mice. Antimicrob Agents Chemother. 2020; 64. https://doi.org/10.1128/AAC.01943-19 PMID: 31767722

42. Polis B, Srikanth KD, Gurevich V, Gil-Henn H, Samson AO. L-Norvaline, a new therapeutic agent against Alzheimer's disease. Neural Regen Res. 2019; 14: 1562-1572. https://doi.org/10.4103/16735374.255980 PMID: 31089055

43. Ganapathy US, Gengenbacher M, Dick T. Epetraborole is Active against Mycobacterium abscessus. Antimicrob Agents Chemother. 2021 [cited 31 Aug 2021]. https://doi.org/10.1128/AAC.01156-21 PMID: 34280020

44. Kim T, Hanh BTB, Heo B, Quang N, Park Y, Shin J, et al. A screening of the mmv pandemic response box reveals epetraborole as a new potent inhibitor against mycobacterium abscessus. Int J Mol Sci. 2021;22. https://doi.org/10.3390/ijms22115936 PMID: 34073006

45. Melnikov S V, Stevens DL, Fu X, Kwok HS, Zhang JT, Shen Y, et al. Exploiting evolutionary trade-offs for posttreatment management of drug-resistant populations. Proc Natl Acad Sci U S A. 2020; 117: 17924-17931. https://doi.org/10.1073/pnas.2003132117 PMID: 32661175

46. Reingewertz TH, Meyer T, McIntosh F, Sullivan J, Meir M, Chang YF, et al. Differential sensitivity of mycobacteria to isoniazid is related to differences in katg-mediated enzymatic activation of the drug. Antimicrob Agents Chemother. 2020;64. https://doi.org/10.1128/AAC.01899-19 PMID: 31767723

47. Rominski A, Roditscheff A, Selchow $P$, Böttger EC, Sander P. Intrinsic rifamycin resistance of Mycobacterium abscessus is mediated by ADP-ribosyltransferase MAB_0591. J Antimicrob Chemother. 2017; 72: 376-384. https://doi.org/10.1093/jac/dkw466 PMID: 27999011

48. Luthra S, Rominski A, Sander P. The Role of Antibiotic-Target-Modifying and Antibiotic-Modifying Enzymes in Mycobacterium abscessus Drug Resistance. Front Microbiol. 2018;9. https://doi.org/10 3389/fmicb.2018.00009 PMID: 29387050

49. Alcaide F, Pfyffer GE, Telenti A. Role of embB in natural and acquired resistance to ethambutol in mycobacteria. Antimicrob Agents Chemother. 1997; 41: 2270-2273. https://doi.org/10.1128/AAC.41.10. 2270 PMID: 9333060

50. Sun Z, Scorpio A, Zhang Y. The pncA gene from naturally pyrarinamide-resistant Mycobacterium avium encodes pyrazinamidase and confers pyrazinamide susceptibility to resistant $\mathrm{M}$. tuberculosis complex organisms. Microbiology. 1997; 143: 3367-3373. https://doi.org/10.1099/00221287-143-10-3367 PMID: 9353938

51. Zhang $\mathrm{Y}$, Scorpio A, Nikaido $\mathrm{H}$, Sun Z. Role of acid $\mathrm{pH}$ and deficient efflux of pyrazinoic acid in unique susceptibility of Mycobacterium tuberculosis to pyrazinamide. J Bacteriol. 1999; 181: 2044-2049. https://doi.org/10.1128/JB.181.7.2044-2049.1999 PMID: 10094680

52. Su H, Xu Y. Application of ITC-based characterization of thermodynamic and kinetic association of ligands with proteins in drug design. Front Pharmacol. 2018; 9: 1-7. https://doi.org/10.3389/fphar.2018. 00001 PMID: 29387012

53. Dubée V, Bernut A, Cortes M, Lesne T, Dorchene D, Lefebvre AL, et al. $\beta$-Lactamase inhibition by avibactam in Mycobacterium abscessus. J Antimicrob Chemother. 2014; 70: 1051-1058. https://doi.org/ 10.1093/jac/dku510 PMID: 25525201

54. Ming XF, Rajapakse AG, Carvas JM, Ruffieux J, Yang Z. Inhibition of S6K1 accounts partially for the anti-inflammatory effects of the arginase inhibitor L-norvaline. BMC Cardiovasc Disord. 2009;9. https:// doi.org/10.1186/1471-2261-9-9 PMID: 19254382

55. Chang C-I, Liao JC, Kuo L. Arginase modulates nitric oxide production in activated macrophages. Am J Physiol. 1998; 274: H324-H348. Available: https://journals.physiology.org/doi/pdf/10.1152/ajpheart. 1998.274.1.H342 PMID: 9458885 
